\title{
The Caustic Ring Model of the Milky Way Halo
}

\author{
L. D. Duffy ${ }^{1, *}$ and P. Sikivie ${ }^{2, \dagger}$ \\ ${ }^{1}$ Theoretical Division, Los Alamos National Laboratory, Los Alamos, NM 87545, USA \\ ${ }^{2}$ University of Florida, Gainesville, FL 32611, USA
}

(Dated: May 28, 2008)

\begin{abstract}
We present a proposal for the full phase space distribution of the Milky Way halo. The model is axially and reflection symmetric and its time evolution is self-similar. It describes the halo as a set of discrete dark matter flows with stated densities and velocity vectors everywhere. We first discuss the general conditions under which the time evolution of a cold collisionless self-gravitating fluid is self-similar, and show that symmetry is not necessary for self-similarity. When spherical symmetry is imposed, the model is the same as described by Fillmore and Goldreich, and by Bertschinger, twenty-three years ago. The spherically symmetric model depends on one dimensionless parameter $\epsilon$ and two dimensionful parameters. We set $\epsilon=0.3$, a value consistent with the slope of the power spectrum of density perturbations on galactic scales. The dimensionful parameters are determined by the Galactic rotation velocity $(220 \mathrm{~km} / \mathrm{s})$ at the position of the Sun and by the age of the Galaxy (13.7 Gyr). The properties of the outer caustics are derived in the spherically symmetric model. The structure of the inner halo depends on the angular momentum distribution of the dark matter particles. We assume that distribution to be axial and reflection symmetric, and dominated by net overall rotation. The inner caustics are rings whose radii are determined in terms of a single additional parameter $j_{\max }$. We summarize the observational evidence in support of the model. The evidence is consistent with $j_{\max }=0.18$ in Concordance Cosmology, equivalent to $j_{\max \text {,old }}=$ 0.26 in Einstein - de Sitter cosmology. We give formulas to estimate the flow densities and velocity vectors anywhere in the Milky Way halo. The properties of the first forty flows at the location of the Earth are listed.
\end{abstract}

PACS numbers: 95.35.+d

*Electronic address: lduffy@lanl.gov

${ }^{\dagger}$ Electronic address: sikivie@phys.ufl.edu 


\section{INTRODUCTION}

It has been established from a variety of observational inputs that approximately $23 \%$ of the energy density of the universe is in the form of "cold dark matter" (CDM) [1]. The CDM particles must be non-baryonic, collisionless, and cold. "Cold" means that their primordial velocity dispersion is small enough that it can be set equal to zero for all practical purposes when discussing the formation of large scale structure. "Collisionless" means that the particles have negligible interactions other than gravity. A central problem in dark matter studies is the question how CDM is distributed in the halos of galaxies, and in particular in the halo of our Milky Way galaxy. Indeed, knowledge of this distribution is essential for understanding galactic dynamics and for predicting signals in direct and indirect searches for dark matter on Earth.

Galactic halos are collisionless fluids and must therefore be described in 6-dimensional phase space. A full description gives the phase space distribution $f(\vec{r}, \vec{v} ; t)$ of the dark matter particles in the halo, i.e. their velocity $(\vec{v})$ distribution at every position $\vec{r}$. $t$ is time. An important simplification occurs in the case of halos built of cold collisionless dark matter because CDM particles lie in phase space on a thin 3-dimensional hypersurface. This fact implies that the velocity distribution is everywhere discrete [2] and that there are surfaces in physical space, called caustics, where the density of dark matter is very large. See Fig. 1 for an illustration. It is argued in ref. [3] that discrete flows and caustics in galactic halos are a generic and robust prediction of cold dark matter cosmology, even after all possible complications and reasons for skepticism have been considered. The reader may wish to consult ref. [3] for background information and a list of references. Discrete flows and caustics are seen in N-body simulations of large scale structure formation when special techniques are used to reach adequate resolution in the relevant regions of phase-space [4, 5, 6].

The caustic ring model is a proposal for the full phase space distribution of the Milky Way halo. The model is axially symmetric, reflection symmetric, and self-similar in its time evolution. The model is an elaboration of the spherically symmetric self-similar model originally proposed by Fillmore and Goldreich [7], and by Bertschinger [8]. Our main purpose in presenting the caustic ring model is to enable its further comparison with observation. The observational evidence which we claim in support of the model thus far is listed in Section IV.

Because the original self-similar halo model [7, 8] is spherically symmetric, one may be tempted to suppose that self-similarity and spherical symmetry are linked somehow, that the latter is required by the former. This is not so, however. In Section II, we discuss the general conditions under which the time evolution of a cold collisionless self-gravitating fluid is self-similar. We show that self-similarity does not require symmetry. We consider three cases: no symmetry, spherical symmetry, and axial symmetry. In each instance, we derive the equations of motion for the reduced variables, and the initial conditions required by self-similarity.

The spherically symmetric infall model is inadequate to describe the inner parts of a cold dark matter halo. Indeed, in a spherically symmetric halo, CDM particles necessarily have vanishing angular momentum. Each particle moves on a radial orbit and passes through the center at each throughfall. The center is then a caustic point where the density $d(r)$

diverges approximately as $\frac{1}{r^{2}}$, where $r$ is galactocentric distance. In realistic halos, the dark matter particles carry angular momentum and therefore miss the center. There are still 
inner caustics in that case [9] but they are spread on surfaces with cusps. The catastrophe structure of the inner caustics [9] depends on the angular momentum distribution of the infalling CDM particles. If the angular momentum distribution is dominated by net overall rotation, the inner caustics are rings (closed tubes) whose cross-section is a section of the elliptic umbilic $\left(D_{-4}\right)$ catastrophe [10,11]. These objects are usually referred to as "caustic rings of dark matter". Figs. 5 and 6 describe the cross-section of a caustic ring of dark matter and indicate what is meant by the ring radius $a$ and by its transverse dimensions $p$ and $q$. In the opposite case, where the velocity distribution is irrotational $(\vec{\nabla} \times \vec{v}=0)$, the inner caustic has a tent-like structure described in detail in ref. [9]. Because there is evidence for caustic rings of dark matter (as opposed to tent-like caustics), in the Milky Way [10, 12] and in other isolated spiral galaxies [13], we assume that the angular momentum distribution is dominated by net overall rotation.

The spherically symmetric self-similar infall model [7, 8] was generalized in ref. [14] to include angular momentum of the infalling particles. The main purpose of that paper was to estimate the densities and speeds of the dark matter flows at a distance of $8.5 \mathrm{kpc}$ from the Galactic center. It was shown in ref. [14] that the angular momentum distribution can be chosen so as to preserve self-similarity. For the sake of convenience, the model of ref. [14] was artificially made spherically symmetric by averaging an actual halo with angular momentum over all orientations. No such averaged halo can be physically realized in cold dark matter cosmology. However, the model of ref. [14] is adequate for its purpose of estimating the speeds and average densities of the dark matter flows at $8.5 \mathrm{kpc}$ from the Galactic center. The model of ref. [14] has inner caustics but they are spread over spheres. The model of ref. [14] is an important precursor to the model presented here. The latter is essentially the former without the averaging over all orientations.

It was assumed in ref. [14] that the universe is Einstein - de Sitter, i.e. spatially flat and matter dominated $\left(\Omega=\Omega_{\mathrm{m}}=1\right)$. This is a convenient assumption when building a selfsimilar model because self-similarity requires the absence of a special time. The Einstein de Sitter cosmology doesn't have a special time and is the only cosmology in which structure formation can be strictly self-similar. Subsequently, the accelerated expansion of the universe was discovered [15] and the Concordance Cosmology $\left(\Omega_{\mathrm{m}}=0.27\right.$ and $\left.\Omega_{\Lambda}=0.73\right)$ established 1]. The Concordance Cosmology is inconsistent with exact self-similarity since it has a special time, namely the time a few Gigayears ago when the universe passed from matter domination to dark energy domination. However, the Milky Way halo was assembled almost entirely before the onset of accelerated expansion, when the universe was well described by $\Omega=\Omega_{\mathrm{m}}=1$ cosmology. The breaking of self-similarity implied by the Concordance Cosmology has only a small effect on the formation of our Galaxy and will be ignored here.

Nonetheless, the discovery of the accelerated expansion of the universe and the advent of Concordance Cosmolgy did affect the self-similar infall model in an important way because they led to a precise determination of the age of the universe. In ref. [14] it was assumed, consistently with Einstein - de Sitter cosmology, that the present age is related to the present Hubble expansion rate $H_{0}$ by $t_{0}=\frac{2}{3 H_{0}}=\frac{1}{h} 6.52 \mathrm{Gyr}$, where $h$ is defined as usual by $H_{0}=h$ $100 \mathrm{~km} /(\mathrm{s} \mathrm{Mpc})$. Since the universe is now known to be 13.7 Gyr old [1, 15], the results of ref. [14] remain valid provided only that $h$ is replaced everywhere in that paper by $\frac{6.52 \mathrm{Gyr}}{t_{0}}$ $=0.476$.

The outline of our paper is as follows. In Section II, we discuss the general conditions under which the time evolution of a cold collisionless self-gravitating fluid is self-similar. We show that spatial symmetry is not necessary for self-similarity. We discuss three cases - no 
symmetry, spherical symmetry and axial symmetry - and obtain the equations of motion for the reduced variables in each case. In Section III, we present the model. We discuss its underlying assumptions, and describe its outer and inner caustics. We provide interpolating formulae to estimate the flow velocities and densities between the inner and outer caustics. We list the estimates for the inner and outer caustic properties which follow from the application of the method of adiabatic invariants to the model. In Section IV, we list the evidence in support of the model. In Section V, we list the densities and velocities of the first forty flows on Earth. Section VI provides a summary.

\section{SELF-SIMILAR COLD DARK MATTER HALOS}

The purpose of this section is to obtain the conditions under which the time evolution of a cold self-gravitating collisionless fluid is self-similar. We show that symmetry is not necessary for self-similarity. First, we discuss self-similar collisionless fluids. Next, we discuss cold collisionless fluids. Thirdly, we discuss cold and self-similar collisionless fluids. In each instance we obtain the relevant equations, without assumptions of symmetry. Finally, we specialize to the cases of spherical and axial symmetry.

\section{A. General conditions for self-similarity}

Let $f(\vec{r}, \vec{v} ; t)$ be the phase space density of a collisionless self-gravitating fluid at time $t$. It satisfies the collisionless Boltzmann equation

$$
\frac{d f}{d t}=\frac{\partial f}{\partial t}+\vec{v} \cdot \frac{\partial f}{\partial \vec{r}}-\vec{\nabla} \Phi \cdot \frac{\partial f}{\partial \vec{v}}=0
$$

where

$$
\Phi(\vec{r}, t)=-G m \int d^{3} r^{\prime} \int d^{3} v \frac{f\left(\vec{r}^{\prime}, \vec{v} ; t\right)}{\left|\vec{r}-\vec{r}^{\prime}\right|}
$$

is the gravitational potential. $m$ is the particle mass.

Let us first discuss simple rescalings of the phase space distribution and evolution rate of such a system. Given that $f(\vec{r}, \vec{v} ; t)$ is a solution of Eqs. (2.1) and (2.2), consider

$$
f^{\prime}(\vec{r}, \vec{v} ; t)=A f\left(\frac{\vec{r}}{R}, \frac{\vec{v}}{V} ; \frac{t}{T}\right) \quad,
$$

where $A, R, V$ and $T$ are constants. It is straightforward to show that $f^{\prime}(\vec{r}, \vec{v} ; t)$ is also a solution provided

$$
V=\frac{R}{T} \quad \text { and } \quad A=\frac{T}{R^{3}} \quad .
$$

Therefore any solution can always be rescaled in two ways: it can be made older (and hence more slowly evolving) and it can be made larger. The age $t$ sets the scale for the density $\rho$ since $G \rho \sim \frac{1}{t^{2}}$. The size $R$ then sets the scale for the velocities $v \sim \frac{R}{t}$, and for the total mass $M \sim \rho R^{3} \sim \frac{R^{3}}{t^{2}}$. Next let us consider self-similar evolution of the fluid, which is an invariance under time-dependent rescalings.

The evolution is called self-similar if, in all aspects, the fluid remains identical to itself except for an overall rescaling of its phase space density by a factor $A(t)$, of its size in the 
spatial directions by a factor $R(t)$ and of its size in the velocity directions by a factor $V(t)$. Thus, the phase space density of a fluid with self-similar evolution satisfies the ansatz

$$
f(\vec{r}, \vec{v} ; t)=A(t) F\left(\frac{\vec{r}}{R(t)}, \frac{\vec{v}}{V(t)}\right)
$$

where $F(\vec{\chi}, \vec{\nu})$ is a rescaled time-independent phase space density. The gravitational potential is then

$$
\Phi(\vec{r}, t)=A(t) R(t)^{2} V(t)^{3} \Psi\left(\frac{\vec{r}}{R(t)}\right)
$$

with

$$
\Psi(\vec{\chi})=-G m \int d^{3} \chi^{\prime} \int d^{3} \nu \frac{F\left(\vec{\chi}^{\prime}, \vec{\nu}\right)}{\left|\vec{\chi}-\vec{\chi}^{\prime}\right|}
$$

Substituting Eqs. (2.5) and (2.6) into Eq. (2.1), one finds that the self-similarity ansatz can be satisfied only if

$$
R(t) \propto t^{\beta} \quad, \quad V(t)=\frac{R(t)}{t} \quad \text { and } \quad A(t)=\frac{t}{R(t)^{3}} \quad .
$$

The rescaled phase space distribution must satisfy

$$
(1-3 \beta) F(\vec{\chi}, \vec{\nu})+(\vec{\nu}-\beta \vec{\chi}) \cdot \frac{\partial F}{\partial \vec{\chi}}+\left((1-\beta) \vec{\nu}-\frac{\partial \Psi}{\partial \vec{\chi}}\right) \cdot \frac{\partial F}{\partial \vec{\nu}}=0
$$

Thus, the evolution of a self-similar self-gravitating collisionless fluid is obtained by choosing $\beta$ and solving simultaneously Eqs. (2.9) and (2.7), with appropriate boundary conditions. Next, we consider a collisionless fluid which is self-gravitating and cold, but not necessarily self-similar.

\section{B. Phase space distribution of cold dark matter}

Cold collisionless dark matter (CDM) particles, such as axions or WIMPs, lie on a thin 3dim. hypersurface in phase space. Indeed, at a sufficiently early time $t_{\text {in }}$, all CDM particles at a given location $\vec{r}$ have the same velocity $\vec{v}_{\text {in }}(\vec{r})$, up to a small species-dependent primordial velocity dispersion $\delta v$. We call the 3-dim. hypersurface the "phase space sheet". The thickness of the phase space sheet is the primordial velocity dispersion $\delta v$. For CDM $\delta v$ is small, i.e. the sheet is thin [11]. The number of particles is huge (approx. $10^{84}$ axions and/or $10^{68}$ WIMPs per galactic halo), so that the sheet is continuous. As time goes on, the sheet folds in phase space.

To describe the evolution of the phase space sheet we label the particles by a continuous parameter $\vec{\alpha}=\left(\alpha_{1}, \alpha_{2}, \alpha_{3}\right)$. The position of particle labeled $\vec{\alpha}$ at time $t$ is $\vec{x}(\vec{\alpha}, t)$. The particle has velocity $\vec{v}(\vec{\alpha}, t)=\frac{\partial \vec{x}}{\partial t}(\vec{\alpha}, t)$. The phase space sheet is the set of points $(\vec{x}(\vec{\alpha}, t), \vec{v}(\vec{\alpha}, t))$ for all $\vec{\alpha}$. It has a time-independent mass density $\frac{d M}{d \alpha^{3}}(\vec{\alpha})$ in parameter space. At any point $\vec{r}$ in physical space, there is a discrete set of flows. The number $N(\vec{r}, t)$ of flows at position $\vec{r}$ and time $t$ is the number of solutions $\vec{\alpha}_{j}(\vec{r}, t), j=1,2 \ldots N$, of the equation $\vec{r}=\vec{x}(\vec{\alpha}, t)$. The $j^{\text {th }}$ flow has velocity

$$
\vec{v}_{j}(\vec{r}, t)=\frac{\partial \vec{x}}{\partial t}\left(\vec{\alpha}_{j}(\vec{r}, t), t\right)
$$


and density

$$
d_{j}(\vec{r}, t)=\frac{d M}{d \alpha^{3}}\left(\vec{\alpha}_{j}(\vec{r}, t)\right) \frac{1}{\left|D\left(\vec{\alpha}_{j}(\vec{r}, t), t\right)\right|}
$$

where

$$
D(\vec{\alpha}, t)=\operatorname{det}\left(\frac{\partial \vec{r}(\vec{\alpha}, t)}{\partial \vec{\alpha}}\right)
$$

Caustics are located where $D\left(\vec{\alpha}_{j}(\vec{r}, t), t\right)=0$ for some $j$. A possible parametrization is $\vec{\alpha}=\vec{r}_{\text {in }}$ where $\vec{r}_{\text {in }}$ is particle position at an arbitrarily chosen but sufficiently early initial time $t_{\text {in }}$. Other parametrizations may be more convenient, however. The flow velocities $\vec{v}_{j}(\vec{r}, t)$ and densities $d_{j}(\vec{r}, t)$ are parametrization independent.

Since the particles are collisionless,

$$
\frac{\partial^{2} \vec{x}(\vec{\alpha}, t)}{\partial t^{2}}=-\left.\vec{\nabla}\left[\Phi(\vec{r}, t)+\Phi_{\mathrm{b}}(\vec{r}, t)\right]\right|_{\vec{r}=\vec{x}(\vec{\alpha}, t)}
$$

where

$$
\Phi(\vec{r}, t)=-G \int d^{3} r^{\prime} \sum_{j=1}^{N\left(\vec{r}^{\prime}, t\right)} \frac{d_{j}\left(\vec{r}^{\prime}, t\right)}{\left|\vec{r}-\vec{r}^{\prime}\right|}=-G \int d^{3} \alpha \frac{d M}{d \alpha^{3}}(\vec{\alpha}) \frac{1}{|\vec{r}-\vec{x}(\vec{\alpha}, t)|}
$$

is the gravitational potential due to dark matter and $\Phi_{\mathrm{b}}$ is the contribution of ordinary matter. The CDM phase space distribution is determined by specifying $\frac{d M}{d \alpha^{3}}(\vec{\alpha})$ and the positions $\vec{r}_{\text {in }}(\vec{\alpha})$ and velocities $\vec{v}_{\text {in }}(\vec{\alpha})$ of the particles at an initial time $t_{\text {in }}$, and solving Eqs. (2.13) and (2.14) simultaneously. $\Phi_{b}(\vec{r}, t)$ has to be supplied by hand or derived in some way.

We wish to apply the above to the construction of galactic halos. Galactic halos grow by accreting more and more of the surrounding dark matter. A convenient choice for the parameters $\vec{\alpha}$ is as follows. Define a sphere of radius $R(t)$ which grows with the halo. $R(t)$ is chosen such that each particle traverses the sphere only once, in the inward direction. The particles are labeled by $\vec{\alpha}=(\theta, \varphi, \tau)$ where $(\theta, \varphi)$ are the spherical coordinates of the point where the particle crosses the sphere and $\tau$ is the time when it does so. For given $R(t)$, the model halo is specified by giving the velocities $\vec{v}_{\text {in }}(\theta, \varphi, \tau)$ of the particles when they cross the sphere and their infall rate per unit solid angle:

$$
\frac{d M}{d \Omega d \tau}(\theta, \varphi, \tau)=\frac{1}{\sin \theta} \frac{d M}{d \theta d \varphi d \tau}(\theta, \varphi, \tau)
$$

Note that the number of degrees of freedom in $\vec{v}_{\text {in }}(\theta, \varphi, \tau)$ is slightly reduced by the freedom to choose $R(t)$. For example, we may choose $R(t)$ such that the particle which is at the intersection of the sphere with the $x$-axis is at first turnaround, for all $t$. In that case, $\vec{v}_{\text {in }}\left(\frac{\pi}{2}, 0, \tau\right) \cdot \hat{x}=0$ for all $\tau$.

The gravitational potential due to dark matter may be separated into two parts: $\Phi=$ $\Phi_{\text {in }}+\Phi_{\text {ex }}$, with

$$
\Phi_{\text {in }}(\vec{r}, t)=-G \int d \Omega \int_{0}^{t} d \tau \frac{d M}{d \Omega d \tau} \frac{1}{|\vec{r}-\vec{x}(\theta, \varphi, \tau ; t)|}
$$

the contribution of particles that have already fallen through the sphere, and

$$
\Phi_{\mathrm{ex}}(\vec{r}, t)=-G \int d \Omega \int_{t}^{\infty} d \tau \frac{d M}{d \Omega d \tau} \frac{1}{|\vec{r}-\vec{x}(\theta, \varphi, \tau ; t)|}
$$


the contribution of particles that have not yet done so. In the spherically symmetric case $\Phi_{\text {ex }}$ can be ignored since it is $\vec{r}$-independent inside the sphere. In the non-spherically symmetric case, to obtain $\Phi_{\mathrm{ex}}$, the equations of motion have to be - in principle - integrated backward in time to determine the positions of the particles outside the sphere. This procedure would then include all the particles in the universe. In practice, the contribution of particles outside the sphere may be given by hand, or ignored.

\section{Self-similar evolution of cold dark matter}

In this subsection, we set $\Phi_{b}=0$ because the presence of ordinary matter generally spoils exact self-similarity by introducing special time and special length scales. However later, when discussing our Milky Way halo model, we will add the baryon contribution to the gravitational potential by hand, maintaining self-similarity, as described in Section III.

We saw in subsection II.A that a self-similar halo grows in physical space as a power law: $R(t) \propto t^{\beta}$. Hence we choose the sphere introduced in subsection II.B to have radius

$$
R(t)=R_{0}\left(\frac{t}{t_{0}}\right)^{\beta}
$$

where $t_{0}$ is an arbitrary reference time. Under the self-similarity assumption, the particle that falls in at time $\tau$ and location $(\theta, \varphi)$ moves in exactly the same way as the particle that falls in at $(\theta, \varphi)$ at any other time $\tau^{\prime}$, except that its trajectory is rescaled in physical space by $\frac{R(\tau)}{R\left(\tau^{\prime}\right)}$ and in time by $\frac{\tau}{\tau^{\prime}}$. Hence there exists a function $\vec{\lambda}(\theta, \varphi ; \xi)$ such that

$$
\vec{x}(\theta, \varphi, \tau ; t)=R(\tau) \vec{\lambda}\left(\theta, \varphi ; \xi=\frac{t}{\tau}\right)
$$

Also the infall rate $\frac{d M}{d \Omega d \tau}(\theta, \varphi, \tau)$ must have a specific time dependence. The total dark matter mass within the sphere of radius $R(t)$ is

$$
\begin{aligned}
M(t) & =\int d \Omega \int_{0}^{t} d \tau \frac{d M}{d \Omega d \tau}(\theta, \varphi, \tau)=m \int_{r<R(t)} d^{3} r \int d^{3} v f(\vec{r}, \vec{v} ; t) \\
& =m A(t) \frac{R^{6}(t)}{t^{3}} \int_{|\vec{\chi}|<1} d^{3} \chi \int d^{3} \nu F(\vec{\chi}, \vec{\nu}) \propto t^{3 \beta-2},
\end{aligned}
$$

where we used Eqs. (2.8). Hence we need

$$
\frac{d M}{d \Omega d \tau}(\theta, \varphi, \tau)=\left.\left(\frac{\tau}{t_{0}}\right)^{3(\beta-1)} \frac{d M}{d \Omega d \tau}\right|_{0}(\theta, \varphi)
$$

Finally, the initial velocities must be of the form

$$
\vec{v}_{\text {in }}(\theta, \varphi, \tau)=\frac{R(\tau)}{\tau} \vec{\nu}_{\text {in }}(\theta, \varphi)
$$

to be consistent with Eq. (2.19).

Substituting Eqs. (2.19) and (2.21) into Eq. (2.14), we obtain

$$
\Phi(\vec{r}, t)=\left(\frac{R(t)}{t}\right)^{2} \Psi\left(\frac{\vec{r}}{R(t)}\right)
$$


with

$$
\Psi(\vec{\chi})=-\left.G\left(\frac{t_{0}}{R_{0}}\right)^{3} \int_{0}^{\infty} \frac{d \xi}{\xi^{3 \beta-1}} \int d \Omega \frac{d M}{d \Omega d \tau}\right|_{0}(\theta, \varphi) \frac{1}{\left|\vec{\chi}-\frac{1}{\xi^{\beta}} \vec{\lambda}(\theta, \varphi ; \xi)\right|}
$$

With $\Phi_{\mathrm{b}}=0$, the equations of motion (2.13) become

$$
\frac{\partial^{2} \vec{\lambda}}{\partial \xi^{2}}=-\xi^{\beta-2} \frac{\partial \Psi}{\partial \vec{\chi}}\left(\vec{\chi}=\frac{\vec{\lambda}(\theta, \varphi ; \xi)}{\xi^{\beta}}\right)
$$

The initial conditions are

$$
\begin{aligned}
\vec{\lambda}(\theta, \varphi ; 1) & =\hat{n}(\theta, \varphi) \\
\frac{\partial \vec{\lambda}}{\partial \xi}(\theta, \varphi ; 1) & =\vec{\nu}_{\text {in }}(\theta, \varphi)
\end{aligned}
$$

where $\hat{n}(\theta, \varphi)$ is the unit vector in the direction $(\theta, \varphi)$. The halo is determined in terms of $\beta, \vec{\nu}_{\text {in }}(\theta, \varphi)$ and $\left.\frac{d M}{d \Omega d \tau}\right|_{0}(\theta, \varphi)$ by solving Eqs. (2.24) and (2.25) with initial conditions (2.26). Again, the freedom to choose $R$ removes one degree of freedom from $\vec{\nu}_{\text {in }}(\theta, \varphi)$. For example, $R$ may be chosen such that $\overrightarrow{\nu_{\text {in }}}\left(\frac{\pi}{2}, 0\right) \cdot \hat{x}=0$.

\section{The spherically symmetric case}

For a halo of cold collisionless particles to be spherically symmetric, the particle orbits must be radial, i.e. each particle has zero angular momentum. Thus

$$
\vec{\nu}_{\text {in }}(\theta, \varphi)=\nu_{\text {in }} \hat{n}(\theta, \varphi) \quad \text {. }
$$

Furthermore $\nu_{\text {in }}$ and $\left.\frac{d M}{d \Omega d \tau}\right|_{0}$ must be independent of $(\theta, \varphi)$. We choose $R(t)$ to be the turnaround radius at time $t$, so that $\nu_{\text {in }}=0$. We have then

$$
\Psi(\chi)=-G\left(\frac{t_{0}}{R_{0}}\right)^{3} \frac{d M}{d t}\left(t_{0}\right) \int_{0}^{\infty} \frac{d \xi}{\xi^{3 \beta-1}} \frac{1}{\left|\chi-\frac{1}{\xi^{\beta}} \lambda(\xi)\right|}
$$

Because of spherical symmetry, $\frac{\partial \Psi}{\partial \chi}(\chi)$ gets contributions only from $\xi$ such that $\frac{1}{\xi^{\beta}} \lambda(\xi)<\chi$. In particular, for $\chi<1$ there is no contribution to $\frac{\partial \Psi}{\partial \chi}$ from the integral over $0<\xi<1$. We may write therefore

$$
\frac{\partial \Psi}{\partial \vec{\chi}}(\vec{\chi})=\hat{\chi} \frac{\pi^{2}}{8} \frac{\mathcal{M}(\chi)}{\chi^{2}}
$$

where

$$
\mathcal{M}(\chi)=G\left(\frac{t_{0}}{R_{0}}\right)^{3} \frac{d M}{d t}\left(t_{0}\right) \frac{8}{\pi^{2}} \int_{1}^{\infty} \frac{d \xi}{\xi^{3 \beta-1}} \Theta\left(\chi-\frac{\lambda(\xi)}{\xi^{\beta}}\right)
$$

The factor $\frac{\pi^{2}}{8}$ is introduced so that $\mathcal{M}(1)=1$ [see Eqs. (2.33) and (2.34) below]. The equation of motion is

$$
\frac{d^{2} \lambda}{d \xi^{2}}=-\frac{\pi^{2}}{8} \frac{\xi^{3 \beta-2}}{\lambda^{2}} \mathcal{M}\left(\chi=\frac{\lambda}{\xi^{\beta}}\right)
$$


Equations (2.30) and (2.31) were first obtained by Fillmore and Goldreich [7]. In terms of the parameter $\epsilon$ which these authors introduced, $\beta=\frac{2}{3}+\frac{2}{9 \epsilon}$. Bertschinger [8] discussed the case $\epsilon=1\left(\beta=\frac{8}{9}\right)$.

Following these authors, consider a spherically symmetric overdensity in an otherwise homogeneous, flat and purely matter dominated universe. Assume the overdensity has a power law profile

$$
\frac{\delta M_{i}}{M_{i}} \propto\left(\frac{1}{M_{i}}\right)^{\epsilon}
$$

where $M_{i}$ is the mass interior to radius $r_{i}$ in the homogeneous universe, and $\delta M_{i}$ is the corresponding overdensity. The exponent $\epsilon$ must be in the range $0<\epsilon \leq 1$. $\epsilon=1$ describes the case where the overdensity is a point mass. It can be shown [7, 8, 14] that the time

evolution of such an overdensity is self-similar with scale factor $R(t) \propto t^{\frac{2}{3}}+\frac{2}{9 \epsilon}$. The mass interior to the turnaround radius $R(t)$ is

$$
M(t)=\frac{\pi^{2}}{8} \frac{R(t)^{3}}{G t^{2}}
$$

Note that $M(t) \propto t^{\frac{2}{3 \epsilon}}$, consistent with Eq. (2.20). Therefore, Eq. (2.30) may be rewritten

$$
\mathcal{M}(\chi)=\frac{2}{3 \epsilon} \int_{1}^{\infty} \frac{d \xi}{\xi^{1+\frac{2}{3 \epsilon}}} \Theta\left(\chi-\frac{\lambda(\xi)}{\xi^{\frac{2}{3}+\frac{2}{9 \epsilon}}}\right) .
$$

We have $\mathcal{M}(1)=1$ since the argument of the $\Theta$-function in Eq. (2.34) is positive for all $\xi$ when $\chi=1$. Thus

$$
M(r, t)=M(t) \mathcal{M}\left(\frac{r}{R(t)}\right)
$$

is the mass interior to radius $r$ at time $t$.

\section{E. The axially symmetric case}

In the axially symmetric case, the model is specified by giving $\beta,\left.\frac{d M}{d \Omega d \tau}\right|_{0}(\alpha)$ and the initial velocity field

$$
\vec{\nu}_{\mathrm{in}}(\theta, \varphi)=\hat{r} \nu_{r}(\alpha)+\hat{\theta} \nu_{\theta}(\alpha)+\hat{\varphi} \nu_{\varphi}(\alpha) \quad,
$$

where $\alpha \equiv \frac{\pi}{2}-\theta$. If reflection symmetry is assumed in addition to axial symmetry, $\left.\frac{d M}{d \Omega d \tau}\right|_{0}$, $\nu_{r}$ and $\nu_{\varphi}$ are even under $\alpha \rightarrow-\alpha$, whereas $\nu_{\theta}$ is odd. One degree of freedom is removed from $\vec{\nu}_{\text {in }}$ by the choice of $R$. For example, we may choose $R$ such that all the particles in the equatorial plane are at first turnaround, in which case $\nu_{r}(0)=0$.

In view of the symmetry, we have

$$
\vec{\lambda}(\theta, \varphi ; \xi)=\hat{z} \lambda_{z}(\alpha, \xi)+\lambda_{\rho}(\alpha, \xi)[\hat{x} \cos \gamma(\alpha, \varphi ; \xi)+\hat{y} \sin \gamma(\alpha, \varphi ; \xi)]
$$

where the $\varphi$ dependence of $\gamma$ is trivial, i.e.

$$
\gamma(\alpha, \varphi ; \xi)=\varphi+\Delta(\alpha, \xi)
$$

The $z$ component of angular momentum is conserved:

$$
j_{z}(\alpha)=\lambda_{\rho}(\alpha, \xi)^{2} \frac{\partial \Delta}{\partial \xi}(\alpha, \xi)=\nu_{\varphi}(\alpha) \cos (\alpha)
$$


$\Delta(\alpha, \xi)$ is determined by solving Eq. (2.39) with the initial condition $\Delta(\alpha, 0)=0$. The equations of motion for the other degrees of freedom are

$$
\begin{aligned}
& \frac{\partial^{2} \lambda_{z}}{\partial \xi^{2}}=-\frac{1}{\xi^{\beta-2}} \frac{\partial \Psi}{\partial \chi_{z}}\left(\vec{\chi}=\frac{\vec{\lambda}}{\xi^{\beta}}\right) \\
& \frac{\partial^{2} \lambda_{\rho}}{\partial \xi^{2}}=-\frac{1}{\xi^{\beta-2}} \frac{\partial \Psi}{\partial \chi_{\rho}}\left(\vec{\chi}=\frac{\vec{\lambda}}{\xi^{\beta}}\right)+\frac{j_{z}(\alpha)^{2}}{\lambda_{\rho}^{3}},
\end{aligned}
$$

with initial conditions

$$
\begin{gathered}
\lambda_{z}(\alpha, 1)=\sin \alpha, \lambda_{\rho}(\alpha, 1)=\cos \alpha \\
\frac{\partial \lambda_{z}}{\partial \xi}(\alpha, 1)=\sin \alpha \nu_{r}(\alpha)-\cos \alpha \nu_{\theta}(\alpha) \\
\frac{\partial \lambda_{\rho}}{\partial \xi}(\alpha, 1)=\cos \alpha \nu_{r}(\alpha)+\sin \alpha \nu_{\theta}(\alpha)
\end{gathered}
$$

\section{THE MODEL}

\section{A. Assumptions and general properties}

To first approximation when discussing its large scale properties, the model is spherically symmetric. So we start with the spherically symmetric self-similar infall model [7, 8] introduced in Section II.D. The dimensionless properties of that model are determined in terms of a single parameter $\epsilon=\frac{2}{9 \beta-6}$. In an average sense, $\epsilon$ is related to the slope of the power spectrum of matter density perturbations [16]. On galactic scales (Mpc), $\epsilon$ is of order $0.3[14]$.

In the spherically symmetric model, the angular momentum of each particle vanishes and all orbits are radial. The particle positions are given by

$$
\vec{r}(\theta, \varphi, \tau ; t)=R(\tau) \hat{n}(\theta, \varphi) \lambda\left(\frac{t}{\tau}\right) \quad,
$$

where $R(t)$ is the turnaround radius at time $t$, and $\tau$ is the time when the particle reached first turnaround. The function $\lambda\left(\xi=\frac{t}{\tau}\right)$ is obtained by solving Eqs. (2.30) and (2.31). This can be done numerically on even a modest computer. Fig. 2 shows the function $\lambda(\xi)$ for $\epsilon=0.3$. Fig. 3 shows the corresponding phase space distribution.

To apply the description to an actual halo, we must provide two dimensionful properties of the halo. Two of the best measured properties of the Milky Way halo are the rotation velocity at the position of the Sun $v_{\text {rot }}\left(r_{\odot}=8.5 \mathrm{kpc}\right)=220 \mathrm{~km} / \mathrm{s}$, and the age $t_{0}=13.7$ Gyr. We will ignore the uncertainties on these two numbers.

Fig. 4 shows the dimensionless rotation velocity squared $\nu^{2}(\epsilon, \chi)$ as a function of dimensionless galactocentric distance $\chi=\frac{r}{R}$ for several values of $\epsilon . \nu(\epsilon, \chi)$ is defined by

$$
v_{\mathrm{rot}}^{2}(r)=\frac{G M(r)}{r}=\nu^{2}\left(\epsilon, \frac{r}{R}\right) \frac{G M}{R}
$$


where $M(r)$ is the mass interior to $r$, and $M=M(R)$. Therefore

$$
\nu^{2}(\epsilon, \chi)=\frac{M(r)}{M} \frac{R}{r}=\frac{\mathcal{M}(\epsilon, \chi)}{\chi} .
$$

$\mathcal{M}(\epsilon, \chi)$ is given by Eq. (2.34). Note that $\nu(\epsilon, 1)=1$. Combining Eqs. (2.33) and (3.2), we have

$$
R=\frac{\sqrt{8}}{\pi} v_{\text {rot }}(r) t \nu^{-1}\left(\epsilon, \frac{r}{R}\right)
$$

The turnaround radius $R$ is obtained by solving Eqs. (3.4) with $t=13.7$ Gyr and $v_{\text {rot }}\left(r_{\odot}\right)$ $=220 \mathrm{~km} / \mathrm{s}$. Table $\llbracket$ gives $R$ and $M$ for various values of $\epsilon$. This is an update of Table $\square$ in ref. 14.

The model is characterized by a set of outer caustics located where the outflows reach their maximum radii $R_{n}(n=1,2,3 \ldots)$ before falling back in. The properties of the outer caustics will be discussed in detail in Section III.B. In addition to these outer caustics, a galactic halo necessarily has inner caustics [9]. In the spherically symmetric model, the inner caustics have all collapsed to the central point. Indeed, since its angular momentum vanishes, each particle passes through the center in the course of each in and out fall. The center is then a singular point, where the density diverges. Fig. 4 shows that, in the spherically symmetric model, the halo contribution to the rotation curve is constant near $r=0$ for $\epsilon \sim$ 0.3 , implying that the density $d(r) \propto \frac{1}{r^{2}}$ as $r \rightarrow 0$.

The spherically symmetric model does not adequately describe the inner parts of the halo. In actual galaxies, the central parts are dominated by baryons and the halo contribution to the rotation curve goes to zero as $r \rightarrow 0$. As pointed out in ref. [14], the depletion of the inner halo, as compared to the prediction of the spherically symmetric self-similar model, is neatly accounted for by the angular momentum that the dark matter particles are expected to carry because angular momentum causes the dark matter particles to avoid the center. Indeed, by constraining the distance scale (effective core radius) over which the inner halo is depleted, one can estimate the average specific angular momentum of the halo particles [14].

When angular momentum is introduced, the inner caustics spread over surfaces with cusps. (The word "cusp" has in this paper the same meaning as in Catastrophe Theory, unrelated to the notion of "cuspy halos".) In ref. [9], the catastrophe structure of the inner caustics was investigated as a function of the angular momentum distribution of the infalling particles. If the angular momentum distribution is dominated by net overall rotation, the inner caustic is a ring (i.e. a closed tube) whose cross-section is a section of the elliptic umbilic $\left(D_{-4}\right)$ catastrophe [11]. We call this type of inner caustic a "caustic ring of dark matter" [10, 11]. Figs. 5 and [6] show such a ring in cross-section. Fig. 5 shows the flow of dark matter in the neighborhood of the caustic, whereas Fig. 6 shows the definition of its radius $a$ and transverse sizes $p$ and $q$. Caustic rings of dark matter are described in more detail in Section III.C below. When the initial velocity distribution is dominated by a curlfree (non rotational) component, the inner caustic has a "tent-like" structure, which may be described qualitatively as a caustic ring of dark matter whose (inner) radius $a$ shrunk to zero while its outer radius $a+p$ was held fixed. See ref. [9] for a detailed description.

As summarized in Section IV, there is evidence for caustic rings of dark matter in the Milky Way and other spiral galaxies. The evidence is for caustic rings, as opposed to the tent-like inner caustics that occur when the angular momentum distribution is irrotational. Hence we assume that the angular momentum distribution of the infalling dark matter is 
dominated by net overall rotation. We assume furthermore that it is axially and reflection symmetric, and consistent with self-similarity. To our previous spherically symmetric description depending on one dimensionless parameter $\epsilon$, we add therefore the dimensionless initial velocity field of Eq. (2.36). The specific angular momentum of the particle at turnaround radius $R(\tau)$ and angular coordinates $(\theta, \varphi)$ is then

$$
\vec{l}(\theta, \varphi, \tau)=\frac{R(\tau)^{2}}{\tau} \vec{j}(\theta, \varphi)
$$

where

$$
\vec{j}(\theta, \varphi)=\hat{n}(\theta, \varphi) \times \vec{\nu}_{\text {in }}(\theta, \varphi)=-\nu_{\varphi}(\alpha) \hat{\theta}+\nu_{\theta}(\alpha) \hat{\varphi} \quad .
$$

The assumption that the initial velocity distribution is dominated by net overall rotation means $\nu_{\varphi} \gg\left|\nu_{r}\right|,\left|\nu_{\theta}\right|$. The simple case of initial rigid rotation is $\nu_{\varphi}(\alpha)=\nu_{\varphi}(0) \cos \alpha$, $\nu_{r}=\nu_{\theta}=0$.

It was mentioned already that, when angular momentum is added to the spherically symmetric infall model, the inner parts of the halo are depleted and the halo contribution to the rotation curve goes to zero when $r \rightarrow 0$, consistent with observation. In the Milky Way and in many other large spiral galaxies, the baryon contribution to the rotation curve is such as to make the curve approximately flat at small $r$, and with approximately the same value at small $r$ as at large $r$. In other words, the baryons and the dark matter "conspire" to keep the rotation velocity approximately constant for all radii where it has been measured. Now, recall that the rotation curve of the spherically symmetric self-similar model is very nearly constant for all $r$ when $\epsilon \sim 0.3$; see Fig. 4. Following ref.[14], we take account of the gravitational force exerted by the baryons in the Galaxy by using the potential of the spherically symmetric model to calculate the motion of the dark matter particles in the axially symmetric model. This should be a good approximation when calculating the properties of low $n$ flows since they are made of particles falling in from distances much greater than the size of the baryonic disk. Furthermore, the caustic rings are made of particles that move in or near the equatorial plane and therefore respond to the same gravitational forces as produce the nearly flat rotation curve of the Galaxy.

In ref. [14], the average value $\bar{j}$ of $j(\theta, \varphi) \equiv|\vec{j}(\theta, \varphi)|$ was estimated to be 0.2 by requiring the halo contribution to the rotation curve to approach zero as $r \rightarrow 0$ over a distance scale (effective core radius) consistent with observations. Subsequently, evidence was found for caustic rings of dark matter in the Milky Way [10, 12]. This evidence is consistent with the maximum value $j_{\max }$ of $j(\theta, \varphi)$ being equal to $0.263 . j_{\max }$ is to be identified with $\nu_{\varphi}(0)$. Although the precise relationship between $\bar{j}$ and $j_{\max }$ depends on the full $j(\theta, \varphi)$-distribution, the values $\bar{j} \sim 0.2$ and $j_{\max } \simeq 0.263$ are consistent with one another. For example, for the simple case of initial rigid rotation, one has $j_{\max }=\frac{4}{\pi} \bar{j}$ and hence $j_{\max }=0.255$ for $\bar{j}=0.2$.

Both $j_{\max } \simeq 0.263$ and $\bar{j} \sim 0.2$ are the outcome of fits to observations in the old $\Omega=\Omega_{\mathrm{m}}=$ 1 cosmology with $H_{0}=70 \mathrm{~km} /(\mathrm{s} \mathrm{Mpc})$ in which the age of our galaxy is 9.31 Gyr. Since we now adopt the age of $13.7 \mathrm{Gyr}$, while keeping the rotation velocity $(220 \mathrm{~km} / \mathrm{s})$ unchanged, all length scales are stretched by the factor $13.7 / 9.31=1.47$. Because the effective core radius is approximately proportional to $\bar{j}$ and the caustic ring radii approximately proportional to $j_{\max }$, the values of these parameters should be reduced by the factor 1.47 compared to the old $\Omega=\Omega_{\mathrm{m}}=1$ model. So the fit to observations yields $\bar{j} \sim 0.14$ and $j_{\max } \simeq 0.179$ in Concordance Cosmology. More precisely, because the caustic ring radii are not exactly proportional to $j_{\max }$, the fit of the model to the evidence in Concordance Cosmology (see Section IV.D) yields $j_{\max }=0.186$, which is the value adopted in this paper. To avoid 
confusion, we write henceforth $j_{\text {max,old }}$ for values in the old $\Omega=\Omega_{\mathrm{m}}=1$ model, and simply $j_{\max }$ for values in the Concordance Cosmology.

For the sake of definiteness, the model presented here assumes $\epsilon=0.3$.

\section{B. Outer caustic spheres}

Caustics are at the boundaries between regions in physical space with differing number of flows. On one side of a caustic there are two more flows than on the other. When an isolated halo is approached from the outside, the local number of flows increases. First, there is one flow, then three, then five, and so on. The boundary between the region with one flow and the region with three flows is the location of the first outer caustic. Likewise, the second outer caustic is at the boundary between the region with three flows and the region with five flows, and so on. See Fig. 1 for an illustration.

The outer caustics are simple fold $\left(A_{2}\right)$ castastrophes located on topological 2-spheres surrounding the galaxy. The density in the 2 extra flows on the inside of an outer caustic diverges on the 2-sphere, with the following characteristic behaviour:

$$
d(x)=\frac{A}{\sqrt{x}} \Theta(x)[1+\mathcal{O}(x)]
$$

where $x$ is the distance to the 2-sphere, measured positively on the inside, $\Theta(x)$ is the step function, and $A$ is a constant which we call the fold coefficient. In the absence of symmetry, $A$ varies with location on the 2 -sphere. The divergence of $d$ as $x \rightarrow 0_{+}$is cut off by the velocity dispersion of the dark matter particles.

Let us emphasize that the existence of outer caustics with the properties listed in the previous paragraph follows exclusively from the existence of cold collisionless dark matter.

\section{Radii $R_{n}$}

Because angular momentum has little influence on the outer caustics, we use the spherically symmetric model to describe their properties and, in particular, to estimate the galactocentric radii $R_{n}$ where they occur and their fold coefficients $A_{n}$. As before, each spherical

shell is labeled by the time $\tau$ when it was at its first turnaround. At time $t$, the radius of a shell is

$$
r(\tau, t)=R(\tau) \lambda\left(\frac{t}{\tau}\right)=\left.R(t) \frac{1}{\xi^{\beta}} \lambda(\xi)\right|_{\xi=\frac{t}{\tau}} \quad,
$$

where we used $R(t) \propto t^{\beta}$. The outer caustics are at radii

$$
R_{n}=R(t) \Lambda\left(\xi_{n}\right)
$$

where the $\xi_{n}$ are the locations of the maxima of $\Lambda(\xi) \equiv \xi^{-\beta} \lambda(\xi)$. 


\section{Fold coefficients $A_{n}$}

From Eqs. (2.34) and (2.35), one may derive the following formula for the density $d(r, t)$ as a sum over flows

$$
d(r, t)=\sum_{j} d_{j}(r, t)=\frac{1}{6 \pi \epsilon} \frac{M(t)}{R(t) r^{2}} \sum_{j} \frac{1}{\xi_{j}(r, t)^{1+\frac{2}{3 \epsilon}}\left|\frac{d \Lambda}{d \xi}\left(\xi_{j}(r, t)\right)\right|}
$$

where the $\xi_{j}(r, t)$ are the solutions of $r=R(t) \Lambda(\xi)$. Near a maximum $\xi_{n}$ of $\Lambda(\xi)$, we have

$$
\begin{aligned}
\Lambda(\xi) & =\Lambda\left(\xi_{n}\right)+\frac{1}{2} \frac{d^{2} \Lambda}{d \xi^{2}}\left(\xi_{n}\right)\left(\xi-\xi_{n}\right)^{2}+\mathcal{O}\left(\xi-\xi_{n}\right)^{3} \\
\frac{d \Lambda}{d \xi} & =+\frac{d^{2} \Lambda}{d \xi^{2}}\left(\xi_{n}\right)\left(\xi-\xi_{n}\right)+\mathcal{O}\left(\xi-\xi_{n}\right)^{2}
\end{aligned}
$$

with $\frac{d^{2} \Lambda}{d \xi^{2}}\left(\xi_{n}\right)<0$. Let us label $j=(n+)$ and $(n-)$ the two flows which form the caustic at $r=R_{n}$. Then (the $t$ dependence is suppressed in the remainder of this subsection)

$$
\xi_{n \pm}(r)-\xi_{n}= \pm \sqrt{\frac{2\left(R_{n}-r\right)}{-R \frac{d^{2} \Lambda}{d \xi^{2}}\left(\xi_{n}\right)}}\left[1+\mathcal{O}\left(\xi_{n \pm}-\xi_{n}\right)\right]
$$

for $r$ close to, but less than, $R_{n}$. Combining Eqs. (3.10) and (3.12), we find

$$
d_{n \pm}(r)=\frac{1}{2} \frac{A_{n}}{\sqrt{R_{n}-r}} \Theta\left(R_{n}-r\right)\left[1+\mathcal{O}\left(\sqrt{R_{n}-r}\right)\right]
$$

with

$$
A_{n}=\frac{1}{3 \pi \epsilon} \frac{M}{\sqrt{2 R} R_{n}^{2}} \frac{1}{\xi_{n}^{1+\frac{2}{3 \epsilon}} \sqrt{-\frac{d^{2} \Lambda}{d \xi^{2}}\left(\xi_{n}\right)}}
$$

The $\mathcal{O}\left(\sqrt{R_{n}-r}\right)$ remainder in Eq. (3.13) has opposite sign for $d_{n+}(r)$ and $d_{n-}(r)$ so that

$$
d_{n}(r)=d_{n+}(r)+d_{n-}(r)=\frac{A_{n}}{\sqrt{R_{n}-r}} \Theta\left(R_{n}-r\right)\left[1+\mathcal{O}\left(R_{n}-r\right)\right]
$$

Table Iists the present $R_{n}$ and $A_{n}$ values in the spherically symmetric infall model with $\epsilon$ $=0.3$, for $n=1,2, \ldots 20$.

\section{Inner caustic rings}

It was mentioned already that dark matter halos necessarily have inner caustics, that the catastrophe structure of the inner caustics depends on the angular momentum distribution

of the infalling dark matter particles [9], and that the inner caustics are rings if, as we assume to be the case, the angular momentum distribution is dominated by net overall rotation. The caustic ring singularity was described in ref. [11] which the reader may wish to consult for background information. 
In the limit of axial and reflection symmetry and where the transverse sizes $p$ and $q$ of a caustic ring are much smaller than its radius $a$, the distribution of dark matter particles in the vicinity of the caustic ring is given by the particle positions

$$
\begin{aligned}
& z(\alpha, \eta)=b \alpha \eta \\
& \rho(\alpha, \eta)=a+\frac{1}{2} u\left(\eta-\eta_{0}\right)^{2}-\frac{1}{2} s \alpha^{2} .
\end{aligned}
$$

We use cylindrical coordinates $(z, \rho, \varphi)$ for position in physical space. Eqs. (3.16) give particle positions at a particular time $t$, which is not shown explicitly. The particles are labeled by parameters $(\alpha, \eta)$. As before, $\alpha \equiv \frac{\pi}{2}-\theta$ where $\theta$ is the polar angle of the particle at the time of its first turnaround. $\eta$ is the time when the particle crosses the $z=0$ plane in the course of its flow through the caustic ring. $t-\eta$ can be thought of as the age of the particle. The particles labeled $(\alpha, \eta)$ form a circle of radius $\rho(\alpha, \eta)$ at a height $z(\alpha, \eta)$ above the $z=0$ plane.

Fig. 5 plots $(\rho(\alpha, \eta), z(\alpha, \eta))$ for continuous $\eta$, and discrete values of $\alpha$. The lines in Fig. 5 are the trajectories of the particles forming the flow, except that positions are plotted as a function of age, whereas for ordinary trajectories position is plotted as a function of time. Let us call the lines of Fig. 5 "age trajectories". Fig. 5 shows that particle density diverges on a closed line which has the shape of a isosceles triangle, but with cusps instead of angles. We call that shape a "tricusp". The location of the tricusp is shown in Fig. [6 for the flow of Fig. 5. It is the envelope of the age trajectories. There are four flows everywhere inside the tricusp and two flows everywhere outside. The caustic, i.e. the surface where the density diverges, lies at the boundary between the region with four flows and the region with two flows.

The physical space density is given by [1]

$$
d(\rho, z)=\left.\frac{1}{\rho} \sum_{j=1}^{N(\rho, z)} \frac{d M}{d \Omega d \eta}(\alpha, \eta) \frac{\cos \alpha}{|D(\alpha, \eta)|}\right|_{\left(\alpha_{j}(\rho, z), \eta_{j}(\rho, z)\right)}
$$

where $\alpha_{j}(\rho, z)$ and $\eta_{j}(\rho, z)$, with $j=1 \ldots N(\rho, z)$, are the solutions of $\rho(\alpha, \eta)=\rho$ and $z(\alpha, \eta)=z \cdot N(\rho, z)$ is the number of flows at position $(\rho, z)$; thus, $N=4$ inside the tricusp and $N=2$ outside. $D(\alpha, \eta)$ is the Jacobian determinant of the map $(\alpha, \eta) \rightarrow(\rho, z)$ :

$$
D(\alpha, \eta) \equiv \operatorname{det}\left(\frac{\partial(\rho, z)}{\partial(\alpha, \eta)}\right)=-b\left[u \eta\left(\eta-\eta_{0}\right)+s \alpha^{2}\right] \quad .
$$

$\frac{d M}{d \Omega d \eta}=\frac{d M}{2 \pi \cos \alpha d \alpha d \eta}$ is the mass falling in per unit solid angle and unit time. The tricusp perimeter is the locus of points $(\rho(\alpha, \eta), z(\alpha, \eta))$ for which $D(\alpha, \eta)=0$. We call $p$ and $q$ the sizes of the tricusp in the $\rho$ and $z$ directions respectively; see Fig. 6. They are given by

$$
p=\frac{1}{2} u \eta_{0}^{2}, \quad q=\frac{\sqrt{27}}{4} \frac{b}{\sqrt{u s}} p
$$

Let us write the velocity of particle labeled $(\alpha, \eta)$ as $\vec{v}(\alpha, \eta)=v_{\varphi}(\alpha, \eta) \hat{\varphi}+v_{\rho}(\alpha, \eta) \hat{\rho}+v_{z}(\alpha, \eta) \hat{z}$. The main component of velocity is in the $\hat{\varphi}$ direction: $v_{\varphi} \simeq v$. In the case of a stationary flow, the velocity components in the $\hat{\rho}$ and $\hat{z}$ directions are

$$
v_{\rho}=-\frac{\partial \rho}{\partial \eta}=-u\left(\eta-\eta_{0}\right) \quad, \quad v_{z}=-\frac{\partial z}{\partial \eta}=-b \alpha \quad .
$$


Here we use the fact that, in case of stationary flow, the particle positions are functions only of their age $t-\eta$. Caustic rings grow in mass and radius on cosmological time scales. Therefore stationarity is not an exact property of caustic rings. However, it is an excellent approximation. In the self-similar model the growth in physical size is tantamount to expansion in all directions by the scale factor $R(t)$. Hence $v_{\rho}$ in Eq. (3.20) should be replaced by $v_{\rho}+\frac{\dot{R}}{R} \rho$ and $v_{z}$ by $v_{z}+\frac{\dot{R}}{R} z$. However the corrections are small, of order $1 \%$ for $n=1$ and less for $n \geq 2$, and will be ignored henceforth. For stationary flow, the speed $v$ is related to $u$ by

$$
u=\frac{v^{2}}{a}
$$

since $u$ is the centrifugal acceleration of the particles at $(z, \rho)=(0, a)$.

Our description of a caustic ring in the limit of axial and reflection symmetry, and where the transverse sizes $p$ and $q$ of the ring are much smaller than its radius $a$, is in terms of six parameters: $a, b, u, \eta_{0}, s$ and $\frac{d M}{d \Omega d \eta}$. We now turn to the self-similar infall model to obtain predictions for many of these parameters. As was discussed in Section III.A, the particles are assumed to fall in the gravitational potential produced by the mass distribution $M(r, t)$ of the spherically symmetric self-similar infall model. The equation of motion is obtained therefore by combining Eqs. (2.25) and (2.29):

$$
\frac{\partial^{2} \vec{\lambda}}{\partial \xi^{2}}=-\hat{\lambda} \frac{\pi^{2}}{8} \xi^{3 \beta-2} \frac{1}{\lambda^{2}} \mathcal{M}\left(\frac{\lambda}{\xi^{\beta}}\right),
$$

and using for $\mathcal{M}(\chi)$ the solution of Eqs. (2.30) and (2.31). The initial conditions are

$$
\vec{\lambda}(\theta, \varphi ; 1)=\hat{n}(\theta, \varphi) \quad, \quad \frac{\partial \vec{\lambda}}{\partial \xi}(\theta, \varphi ; 1)=\vec{\nu}_{\text {in }}(\theta, \varphi)
$$

where $\vec{\nu}_{\text {in }}(\theta, \varphi)$ is the initial velocity defined in Eq. (2.36) $)$.

\section{Radii $a_{n}$}

The radius $a_{n}$ of the $n^{\text {th }}$ caustic ring is the distance of closest approach to the galactic center of the particles in the equatorial plane for the $n^{\text {th }}$ in and out flow. To obtain the $a_{n}$ we solve Eq. (3.22) with $\alpha=\theta-\frac{\pi}{2}=0$. By reflection symmetry, $\nu_{\theta}(0)=0$. Also, $\nu_{r}(0)=0$ by definition of the turnaround radius $R$. Eq. (3.22) implies conservation of angular momentum. We have

$$
\lambda^{2}(\xi) \frac{d \varphi}{d \xi}=\nu_{\varphi}(0)
$$

for the particles in the equatorial plane. The quantity $\nu_{\varphi}(0)$ was called $j_{\max }$ in previous publications. For the sake of consistency, we continue to use this name here. The equation of motion for the radial degree of freedom is then

$$
\frac{d^{2} \lambda}{d \xi^{2}}=+\frac{j_{\max }^{2}}{\lambda^{3}}-\frac{\pi^{2}}{8} \xi^{3 \beta-2} \frac{1}{\lambda^{2}} \mathcal{M}\left(\frac{\lambda}{\xi^{\beta}}\right) .
$$

The radial coordinate of the particles that reached first turnaround at time $\tau$ is

$$
r(\tau, t)=R(\tau) \lambda\left(j_{\max }, \frac{t}{\tau}\right)=\left.R(t) \frac{1}{\xi^{\beta}} \lambda\left(j_{\max }, \xi\right)\right|_{\xi=\frac{t}{\tau}}
$$


where $\lambda\left(j_{\max }, \xi\right)$ is the solution of Eq. (3.25) with initial conditions: $\lambda\left(j_{\max }, 1\right)=$ $1, \frac{d \lambda}{d \xi}\left(j_{\max }, 1\right)=0$. The caustic ring radii $a_{n}$ are

$$
a_{n}=R(t) \Lambda\left(j_{\max }, \xi_{n}^{\prime}\right)
$$

where the $\xi_{n}^{\prime}$ are the locations of the minima of $\Lambda\left(j_{\max }, \xi\right) \equiv \xi^{-\beta} \lambda\left(j_{\max }, \xi\right)$. The second column of Table III lists the $a_{n}$ for $n=1,2, \ldots 20$, in the $\epsilon=0.3$ model with $j_{\max }=0.186$. This value of $j_{\max }$ in the Concordance Cosmology was determined from a fit of the $a_{n}$ to a set of rises in the Milky Way rotation curve, as discussed in Section IV.

\section{Accelerations $u_{n}$}

The particles at $(z, \rho)=\left(0, a_{n}\right)$ are moving in the $\hat{\varphi}$ direction with speed $v_{n}=\frac{l_{n}}{a_{n}}$ where

$$
l_{n}=j_{\max } \frac{R^{2}\left(\tau_{n}\right)}{\tau_{n}}=j_{\max } \frac{R^{2}(t)}{t} \frac{1}{\xi_{n}^{\prime 2 \beta-1}}
$$

is their specific angular momentum. Combining Eqs. (3.27) and (3.28), we obtain

$$
v_{n}=\frac{R(t)}{t} j_{\max } \frac{1}{\xi_{n}^{\prime \beta-1} \lambda\left(j_{\max }, \xi_{n}^{\prime}\right)} .
$$

The $u_{n}$ parameters are then

$$
u_{n}=\frac{v_{n}^{2}}{a_{n}}
$$

The speeds $v_{n}$ are listed in the third column of Table III for $\epsilon=0.3$ and $j_{\max }=0.186$. Note that the $v_{n}$ only have a weak dependence on $j_{\max }$ because the denominator in the RHS of Eq. (3.29) is proportional to $j_{\max }$ in the small $j_{\max }$ limit.

$$
\text { 3. Infall rates }\left.\frac{d M}{d \Omega d \eta}\right|_{n}
$$

The infall rates are obtained by noting that $M(t) \propto t^{\frac{2}{3 \epsilon}}$ and assuming that the infall is isotropic. We have

$$
\left.\frac{d M}{d \Omega d \eta}\right|_{n}=\frac{d M}{d \Omega d \tau}\left|\frac{d \tau}{d \eta}\right|_{n}=\frac{1}{4 \pi} \frac{2}{3 \epsilon} \frac{M\left(\tau_{n}\right)}{\tau_{n}} \frac{\tau_{n}}{t}=\frac{1}{6 \pi \epsilon} \frac{M(t)}{t} \frac{1}{\xi_{n}^{\prime \frac{2}{3 \epsilon}}}
$$

That $\left.\frac{d \tau}{d \eta}\right|_{n}=\left.\frac{d \tau}{d t}\right|_{n}=\frac{\tau_{n}}{t}$ follows from self-similarity since, in the absence of any time scale, $\tau_{n}$ and $t$ must be proportional to one another. The fourth column of Table III shows the $\left.\frac{d M}{d \Omega d \eta}\right|_{n}$ values in the model. 


$$
\text { 4. } b_{n}, \eta_{0, n}, s_{n}
$$

The remaining parameters $b_{n}, \eta_{0, n}$ and $s_{n}$ are also predicted by the self-similar infall model but are related to more detailed properties of $\overrightarrow{\nu_{\text {in }}}$ near $\alpha=0$. We have [1]

$$
b_{n}=v_{n}\left[\cos \delta_{n}(0)+\phi^{\prime}(0) \sin \delta_{n}(0)\right]
$$

and

$$
\eta_{0, n}=\frac{a_{n}}{v_{n}} \frac{\phi^{\prime}(0) \cos \delta_{n}(0)-\sin \delta_{n}(0)}{\phi^{\prime}(0) \sin \delta_{n}(0)+\cos \delta_{n}(0)}
$$

where

$$
\phi^{\prime}(0)=-\frac{1}{j_{\max }} \frac{d \nu_{\theta}}{d \alpha}(0)
$$

and

$$
\delta_{n}(0)=-\frac{\pi}{2}+j_{\max } \int_{1}^{\xi_{n}^{\prime}} \frac{d \xi}{\lambda(\xi)^{2}}
$$

Ref. [11] gives also a formula for the $s_{n}$ involving higher derivatives of $\nu_{\theta}(\alpha)$ and $\nu_{\varphi}(\alpha)$ at $\alpha=0$. Generally, $s_{n}$ and $a_{n}$ are of the same order of magnitude.

\section{Interpolating between inner and outer caustics}

The model predicts the full phase space distribution of the halo at all times, i.e. it predicts the number $N(\vec{r}, t)$ of flows at all locations, the flow densities $d_{j}(\vec{r}, t)$ and the flow velocities $\vec{v}_{j}(\vec{r}, t)$. To obtain these quantities one uses, in principle, the following procedure. Obtain $\vec{\lambda}(\theta, \varphi ; \xi)$ by solving Eqs. (3.22) with initial conditions (3.23) for all $(\theta, \varphi)$. Then find the solutions $(\theta, \varphi, \tau)_{j}, j=1,2 \ldots N$, of

$$
\vec{r}=R(t)\left(\frac{\tau}{t}\right)^{\beta} \vec{\lambda}\left(\theta, \varphi ; \frac{t}{\tau}\right)
$$

The number of solutions $N$ is the number of flows at location $\vec{r}$ at time $t$. The velocities of the flows are

$$
\vec{v}_{j}(\vec{r}, t)=\frac{R(t)}{t}\left(\frac{\tau_{j}}{t}\right)^{\beta-1} \frac{\partial \vec{\lambda}}{\partial \xi}\left(\theta_{j}, \varphi_{j} ; \xi=\frac{t}{\tau_{j}}\right)
$$

Their densities are

$$
d_{j}(\vec{r}, t)=\left.\frac{d M}{d \Omega d \tau}(\tau) \frac{\sin \theta}{\left|\operatorname{det}\left(\frac{\partial(x, y, z)}{\partial(\theta, \varphi, \tau)}\right)\right|}\right|_{\left(\theta_{j}, \varphi_{j}, \tau_{j}\right)}
$$

It is certainly possible to carry out this procedure in practice, but it would be laborious. We note that the underlying formalism keeps track of all particles at all times. Since the particles are indistinguishable, this is far more information than the full phase space distribution at all times. Many practical tests of the model are sensitive only to the phase space distribution.

The purpose of this section is to give approximate formulas for the full phase space distribution today, based upon the smooth interpolation between inner and outer caustics. The generalization to other times is straightforward since all lengths scale as $t^{\beta}$, all velocities as $t^{\beta-1}$ and all densities as $t^{-2}$. 
At an arbitrary location $\vec{r}$ which is not within the tricusp tube of a caustic ring, the number of flows is $N(r)=1+2 \sum_{n} \Theta\left(R_{n}-r\right)$ where $\Theta(x)$ is the step-function. For example at $r=130 \mathrm{kpc}$, there are 9 flows because four outer caustics have radius larger than 130 kpc; see Table II. If $\vec{r}$ is within the tricusp tube of a caustic ring, the number of flows is $N(r)=3+2 \sum_{n} \Theta\left(R_{n}-r\right)$ because there are two more flows inside the tricusp tube of a caustic ring than outside.

For all $r$ the flow speeds $v_{n \pm}(r)$ can be read off from Fig. 3. This neglects the effect of angular momentum on flow speed, a small effect. The flow speeds can also be estimated more crudely by using the formula

$$
v_{n \pm}(r)=v_{\text {rot }} \sqrt{2 \ln \left(\frac{R_{n}}{r}\right)} .
$$

Eq. (3.39) would follow from energy conservation in the static gravitational potential $\Phi(r)=$ $v_{\text {rot }}^{2} \ln \left(\frac{1}{r}\right)$. Because the actual potential is time-dependent, energy conservation is only approximate. It is least reliable for $n=1$ since the gravitational potential is changing appreciably on the time scale of the first infall.

To estimate the density of the $n^{\text {th }}$ in and out flows one may use

$$
d_{n \pm}(r)=\left.\frac{1}{r^{2}} \frac{1}{v_{n \pm}(r)} \frac{d M}{d \Omega d t}\right|_{n \pm}(r)
$$

provided $r>>a_{n}$. Eq. (3.40) expresses particle number conservation in the spherically symmetric case. The infall rates are given by

$$
\left.\frac{d M}{d \Omega d t}\right|_{n \pm}(r)=\frac{1}{6 \pi \epsilon} \frac{M}{t} \frac{1}{\xi_{n \pm}(r)^{\frac{2}{3 \epsilon}}}
$$

where the $\xi_{n \pm}(r)$ are the solutions of $r=R(t) \Lambda(\xi)$. Eqs. (3.41) is obtained through the same steps as Eq. (3.31). The infall rates are smooth functions of $\xi$ and may be obtained by interpolating between their values at the inner caustics, given in Table III.

In the spherically symmetric case, the flow equations near radius $r$ are

$$
z(\alpha, \eta)=v(r) \eta \sin \alpha, \quad \rho(\alpha, \eta)=v(r)|\eta| \cos \alpha
$$

where $v(r)$ is the speed of the flow at radius $r$. As before, $\eta$ is the time when the particle crosses the $z=0$ plane. These equations are valid for $r>>a$ where $a$ is the radius of the inner caustic made by that flow. Close to the inner caustic, the flow is described by Eqs. (3.16). Comparing Eqs. (3.16) and (3.42) suggests an interpolating formula for the case $b=v$ and $s=a$ :

$$
z=v \eta \sin \alpha, \quad \rho=\sqrt{a^{2}+v^{2}\left(\eta-\eta_{0}\right)^{2}} \cos \alpha .
$$

Indeed, since $u=\frac{v^{2}}{a}$, Eqs. (3.43) reduce to Eqs. (3.16) for small $\eta$ and small $\alpha$ when $b=v$ and $s=a$, and reduce to Eqs. (3.42) for large $\eta$ and all $\alpha$. To accomodate $b \neq v$ and/or $s \neq a$, one may generalize Eqs. (3.43) to

$$
z=v \eta \sin \left(g_{z}(\eta) \alpha\right) \quad, \quad \rho=\sqrt{a^{2}+v^{2}\left(\eta-\eta_{0}\right)^{2}} \cos \left(g_{\rho}(\eta) \alpha\right)
$$

where $g_{z}(\eta)$ and $g_{\rho}(\eta)$ are smooth functions which approach respectively $\frac{b}{v}$ and $\sqrt{\frac{s}{a}}$ when $\eta \rightarrow 0$, but approach one when $\eta>>\eta_{0}$. 
Sufficiently far from a caustic ring, one may choose to neglect its transverse dimensions $p$ and $q$. This is done by setting $\eta_{0}=0$. In that limit, Eqs. (3.43) may be easily inverted to obtain $\eta(z, \rho)$ and $\alpha(z, \rho)$. One can then express the density and velocity fields directly in terms of position [11]:

$$
\begin{aligned}
& v_{z}=\mp \frac{v}{a} \sqrt{\frac{1}{2}\left(a^{2}-r^{2}+\sqrt{\left(r^{2}-a^{2}\right)^{2}+4 a^{2} z^{2}}\right)} \\
& v_{\rho}=\mp \operatorname{sign}(z) \frac{v}{2 a^{2} \rho} \sqrt{\frac{1}{2}\left(r^{2}-a^{2}+\sqrt{\left(r^{2}-a^{2}\right)^{2}+4 a^{2} z^{2}}\right)}\left(r^{2}+a^{2}-\sqrt{\left(r^{2}-a^{2}\right)^{2}+4 a^{2} z^{2}}\right) \\
& v_{\varphi}=+\sqrt{v^{2}-v_{z}^{2}-v_{\rho}^{2}} \\
& d_{+}=d_{-}=\frac{1}{v} \frac{d M}{d \Omega d \tau} \frac{1}{\sqrt{\left(r^{2}-a^{2}\right)^{2}+4 a^{2} z^{2}}},
\end{aligned}
$$

where the $\mp$ signs are for the down and up flows.

\section{E. Adiabatic approximation}

Except during the first couple throughfalls, the gravitational potential seen by a dark matter particle is slowly varying on the oscillation time scale of its radial coordinate. By exploiting the adiabatic invariant for the radial motion of the particle, it is possible to estimate its trajectory without resorting to numerical integration. This method was used in ref. [7] to show that the halo density $d \propto \frac{1}{r^{2}}$ as $r \rightarrow 0$ in the spherical infall model with $\epsilon<\frac{2}{3}$. It was further used in ref. [14] to obtain estimates of the densities and speeds of the flows at our distance from the galactic center. The adiabatic method gives predictions for the quantities of interest here. We state these predictions without proof, leaving their derivation to a future publication.

For the outer caustics, the adiabatic method predicts

$$
\begin{aligned}
\xi_{n} & =\left[\frac{4}{\sqrt{\pi}} \frac{4+3 \epsilon}{9 \epsilon} n+1\right]^{\frac{9 \epsilon}{4+3 \epsilon}} \\
R_{n} & =R(t) \xi_{n}^{-\frac{4+3 \epsilon}{9 \epsilon}} \\
A_{n} & =\frac{2}{3 \pi^{2} \epsilon} \frac{M}{R^{\frac{5}{2}}} \xi_{n}^{\frac{1}{2}} .
\end{aligned}
$$

To obtain these results, one assumes that the adiabatic invariant for radial motion is constant and that the potential is exactly logarithmic, i.e. $\Phi(r, t)=-v_{\text {rot }}(t)^{2} \ln (r)$.

For the inner caustics, the adiabatic method predicts

$$
\begin{aligned}
& \xi_{n}^{\prime}=\left[\frac{4}{\sqrt{\pi}} \frac{4+3 \epsilon}{9 \epsilon}\left(n-\frac{1}{2}\right)+1\right]^{\frac{9 \epsilon}{4+3 \epsilon}} \\
& a_{n}=\frac{R(t)}{\gamma}\left(\xi_{n}^{\prime}\right)^{-\frac{4+3 \epsilon}{9 \epsilon}} \\
& v_{n}=\frac{R(t)}{t} \gamma j_{\max }
\end{aligned}
$$


where $\gamma$ is given in terms of $j_{\max }$ by

$$
j_{\max }=\frac{\pi}{2} \sqrt{\frac{\ln (\gamma)}{\gamma^{2}-1}} .
$$

To obtain Eqs. (3.47), one makes the same assumptions as for Eqs. (3.46) plus the assumption that $j_{\max }$ is small.

Comparison with the values listed in Tables [I] and [II shows that the adiabatic method agrees with the results of numerical integration usually to within $10 \%$ or $20 \%$. The overall agreement may be surprising considering that the first couple of throughfalls are not in the adiabatic regime, and that an exactly flat rotation curve is assumed to derive the predictions of the adiabatic approximation. Fig. 4 shows that the rotation curve of the self-similar infall model is only approximately flat, even for $\epsilon \simeq 0.3$.

The adiabatic method is useful because it provides a check on the results of numerical integration. It explains patterns in the numerical results that are otherwise mysterious. In particular, it predicts that $v_{n}$ is $n$-independent and that $R_{n}$ and $a_{n} \propto \frac{1}{n}$ for large $n$; see Eqs. (3.46) and (3.47). These properties are descriptve of the values listed in Table II and III. Finally, Eqs. (3.46) and (3.47) enable quick estimates of all model properties for $\epsilon \neq 0.3$.

\section{OBSERVATIONAL EVIDENCE}

In this section, we list a set of observations which are consistent with the caustic ring halo model, and thus lend it support.

\section{A. Flat rotation curves}

The model predicts the Galactic rotation curve at large radii $r$ all the way up to the turnaround radius $R$. The rotation curves for various values of $\epsilon$ are shown in Fig. 4 in dimensionless units. They are nearly flat when $\epsilon \sim 0.3$, implying that the density $d$ is approximately proportional to $\frac{1}{r^{2}}$ at large $r$ all the way up to the turnaround radius $R$, whereabouts $d$ reaches the average value of the cosmological dark matter density. In contrast, the density profiles predicted by computer simulations [17] behave as $d(r) \propto \frac{1}{r^{3}}$ at large $r$, implying that the rotation velocity decreases as $\frac{1}{\sqrt{r}}$.

The rotation cuve of the Milky Way is consistent with being flat up to the largest radii, of order $20 \mathrm{kpc}$, where it has been measured. More importantly, the rotation curves of spiral galaxies in general are flat at large radii up to the largest radii, of order $70 \mathrm{kpc}$, where they have been measured. A collection of extended well measured rotation curves was published in refs. [18, 19].

Furthermore, studies of the dynamics of satellite galaxies [20] provide evidence that the $d \propto r^{-2}$ behavior extends to $r \sim 200 \mathrm{kpc}$. Weak lensing studies of the distortion of galaxy shapes by the gravitational fields of foreground galaxies [21] provide evidence that the behavior extends to $r \sim 370 \mathrm{kpc}$. 


\section{B. Effective core radii}

At small $r$, the Milky Way rotation curve is well accounted for by the baryonic matter in the Galactic bulge and disk. So, the halo contribution to the rotation curve should be suppressed at small radii and should vanish at $r=0$ [22]. The self-similar model generalized to include angular momentum of the dark matter particles [14] does have that property. In

ref. [14], the 'effective core radius' $r_{\mathrm{c}, \text { eff }}$ of a galactic halo was defined as the radius at which the halo contribution to the rotation velocity squared is half its value, $v_{\text {rot }}^{2}$, at large $r$ where the halo dominates. $r_{\mathrm{c}, \mathrm{eff}}$ is proportional to the average amount of dimensionless angular momentum $\bar{j}$. The value of $r_{\mathrm{c}, \text { eff }}$ for the Milky Way, estimated by modeling the bulge and disk contributions to rotation curve, implies $\bar{j}_{\text {old }} \sim 0.2[14]$.

\section{Combined rotation curve}

Table III gives the model predictions for the caustic ring radii $a_{n}$. The predictions may be approximated by

$$
a_{n} \simeq \frac{40 \mathrm{kpc}}{n}\left(\frac{v_{\mathrm{rot}}}{220 \mathrm{~km} / \mathrm{s}}\right)\left(\frac{j_{\mathrm{max}, \mathrm{old}}}{0.27}\right)
$$

for $\epsilon=0.3$. For other values of $\epsilon$ in the range 0.2 to 0.35 , the $a_{n} \propto \frac{1}{n}$ approximate law holds also, with the overall scale being $\epsilon$-dependent.

Since the caustic rings are in or near the galactic plane, they cause bumps in the rotation curve at $r \simeq a_{n}$. Galactic rotation curves have bumps for many reasons unrelated to caustics. However the bumps caused by caustics have a special pattern, Eq.(4.1). One may hope to find evidence for this pattern in a statistical analysis of many galactic rotation curves.

In refs. [18, 19] 32 extended and well measured galactic rotation curves were selected under the criteria that each is an accurate tracer of the radial force law and that it extends far beyond the edge of the luminous disk. The data set of refs. [18, 19] was analyzed as follows [13]. For each rotation curve, the radial variable was rescaled according to

$$
r \rightarrow \tilde{r}=r\left(\frac{220 \mathrm{~km} / \mathrm{s}}{v_{\text {rot }}}\right),
$$

where $v_{\text {rot }}$ is the rotation velocity read off from that particular curve. The data points for $\tilde{r}<10 \mathrm{kpc}$ were deleted to remove the effect of the luminous disk. The 32 rotation curves were then co-added to make a combined rotation curve.

There are two peaks in the combined rotation curve, one near 40 and one near $20 \mathrm{kpc}$, with significance of $3.0 \sigma$ and $2.6 \sigma$, respectively. No explanation has been given for the occurence of the two peaks other than that they are the effect of the $n=1$ and $n=2$ caustic rings of dark matter. The result suggests not only the existence of caustic rings of dark matter distributed according to Eq. (4.1), but also that the $j_{\max }$ distribution of the 32 galaxies in the data set is peaked near $j_{\text {max old }}=0.27$ for $\epsilon=0.3$.

That the $j_{\max }$ distribution is peaked near $j_{\text {max old }}=0.27$ was not a prediction of the self-similar infall model but it is certainly an interesting outcome of the analysis. It was mentioned in the previous subsection that the average magnitude $\bar{j}$ of the dimensionless angular momentum of the Milky Way had been determined to be $\bar{j}_{\text {old }} \sim 0.2$ from an estimate of the effective core radius of its halo. For a given $j$ distribution on the turnaround sphere, $j_{\max }$ and $\bar{j}$ are related. The simplest $j$ distribution is that of a rigidly rotating sphere 
$\left[j(\theta)=j_{\max } \sin \theta\right]$, in which case $j_{\max }=\frac{4}{\pi} \bar{j}$. If we adopt this model, the estimate of the effective core radius of the Milky Way implies that its $j_{\text {max old }} \sim \frac{4}{\pi} 0.2=0.255$. This is close to the peak in the $j_{\max }$ distribution of the 32 external galaxies.

Finally, we mention that the rotation curve of NGC3198, which may be the best measured in the set [18, 19], by itself shows three faint bumps at radii consistent with Eq. (4.1) and $j_{\text {max }, \text { old }}=0.28[10]$.

\section{Milky Way rotation curve}

The effect of a caustic ring in the plane of a galaxy upon its rotation curve was analyzed in ref.[11]. A caustic ring of radius $a$ and width $p$ produces a rise in the rotation curve which starts with an upward kink at $r_{1}=a$ and ends with a downward kink at $r_{2}=a+p$. The two discontinuities are a direct consequence of the fact that the dark matter density associated with the caustic ring diverges at $r=a$ and $r=a+p$.

For technical reasons, the Milky Way rotation curve is measured more precisely at $r<r_{\odot}$, where $r_{\odot}$ is our own galactocentric distance, than at $r>r_{\odot}$. We assume the standard value $r_{\odot}=8.5 \mathrm{kpc}$. The most detailed inner Galactic rotation curve, that we are aware of, was derived [23] from the Massachusetts-Stony Brook North Galactic Plane CO survey [24]. It has a series of sharp rises between 3 and $8.5 \mathrm{kpc}$ [12]. Strikingly, each rise starts with an upward kink and ends with a downward kink, as expected for rises caused by caustic rings of dark matter. Where each rise starts $\left(r=r_{1}\right)$ and ends $\left(r=r_{2}\right)$, the slope of the rotation curve changes abruptly, from one data point to the next.

Eq. (4.1) predicts ten caustic rings between 3 and $8.5 \mathrm{kpc}$, assuming $j_{\text {max,old }} \simeq 0.255$, as was inferred above from the effective core radius of the Milky Way halo. Allowing for ambiguities in identifying rises, the number of rises in the rotation curve [23] between 3 and $8.5 \mathrm{kpc}$ is in fact ten plus or minus one. The radii where the observed rises start $\left(r_{1, n}\right)$ and end $\left(r_{2, n}\right)$ are listed in Table IV under entries $n=5,6 \ldots 14$. The self-similar infall model predictions for the caustic ring radii were fitted to the rises [12]. For a given value of $\epsilon$, this is a one parameter fit, $j_{\max }$ being the only free parameter. For $\epsilon=0.3$, the best fit occurs for $j_{\text {max old }}=0.263$. This is consistent with the estimate $\bar{j}_{\text {old }} \sim 0.2$ obtained from fitting the effective core radius of the Milky Way (see subsections IV.B and IV.C). The root mean square relative deviation between the fitted caustic ring radii $a_{n}$ and the radii $r_{1 n}$, where the rises in the rotation curve start with an upward kink, is $3.1 \%$.

As explained in Section III.A, $j_{\text {max }, \text { old }}=0.263$ in Einstein - de Sitter cosmology corresponds to $j_{\max } \simeq 0.179$ in Concordance Cosmology. We repeated the fit of the calculated $a_{n}$ to the observed $r_{1, n}$ in Concordance Cosmology and obtained $j_{\max }=0.186$, which is the value adopted here. The slight shift in the fitted value of $j_{\max }$ compared to ref. [12] is due to the fact that the $a_{n}$ are only approximately proportional to $j_{\max }$, so that the overall increase in length scales by the factor 1.47 (see Section IIA) between the Einstein - de Sitter and Concordance cosmologies is only approximately compensated for by dividing $j_{\max }$ by 1.47 .

As already mentioned, the rotation curve of the Milky Way is much less well measured for $r>r_{\odot}$. It does however have a prominent rise between 12.7 and $13.7 \mathrm{kpc}$, where Eq.(4.1) predicts the $n=3$ caustic ring to lie. See, for example, the Milky Way rotation curve published in ref. [25]. 


\section{E. Triangular feature in IRAS map}

The gravitational fields of caustic rings of dark matter may leave imprints upon the distribution of ordinary matter. Looking tangentially to a caustic ring from a vantage point in the plane of the ring, one may recognize the tricusp shape of the $D_{-4}$ catastrophe. The IRAS map of the galactic disk in the direction of galactic coordinates $(l, b)=\left(80^{\circ}, 0^{\circ}\right)$ shows a triangular shape which is strikingly suggestive of the cross-section of a caustic ring [12]. The relevant IRAS maps are posted at http://www.phys.ufl.edu/ sikivie/triangle/ . The triangular shape is correctly oriented with respect to the galactic plane and the galactic center. To an extraordinary degree of accuracy it is an isosceles triangle with axis of symmetry parallel to the galactic plane, as is expected for a caustic ring whose transverse dimensions are small compared to its radius. Moreover its position is consistent with the position of the rise in the rotation curve, between 8.28 and $8.43 \mathrm{kpc}$, caused by the caustic ring of dark matter nearest to us.

\section{F. The Monoceros Ring of stars}

The model predicts that the second caustic ring of dark matter $(n=2)$ lies in the Galactic plane at radius $a_{2} \simeq 20 \mathrm{kpc}$. After this prediction was made [10], a ring of stars, named the "Monoceros Ring", was discovered in the Galactic plane at $r \simeq 20 \mathrm{kpc}[26$ ]. It is shown in ref. [27] that the Monoceros Ring of stars is the plausible outcome of the presence of the second caustic ring of dark matter. In particular, it was shown that the adiabatic modification of disk star orbits by the gravitational field of the caustic ring causes an order $100 \%$ enhancement of the density of disk stars at the location of the caustic ring. It was also shown that viscous forces drive the gas in the neighborhood of the caustic ring towards the caustic ring radius $a_{2}$ which may therefore be a site of enhanced star formation.

\section{THE FLOW DENSITIES AND VELOCITIES ON EARTH}

Our study of the phase space structure of the Milky Way halo is motivated in large part by the ongoing searches for dark matter on Earth, using axion [28] and WIMP [29] detectors. The signal in such detectors depends on the velocity distribution of dark matter in the solar neighborhood. The caustic ring halo model predicts that most of the local dark matter is in discrete flows, and predicts the velocity vectors and densities of the flows. Table $\mathrm{V}$ lists the properties of the first forty flows at the Earth's location in the Galaxy. Earlier versions of this table appeared in refs. [30] and [31]. The purpose of this section is to describe how the estimates of Table $\mathrm{V}$ were obtained and to comment on the uncertainties.

As described earlier, the flows come in pairs labeled $(n, \pm)$. The flow velocities are stated in a reference frame which is attached to the Galaxy but which is not rotating relative to the faraway universe. The flow speeds (column 2) follow from the kinetic energy acquired by the particles while they fall in the growing gravitational potential well of the Galaxy. We calculate the speeds at the location of the Sun, at $r_{\odot} \equiv 8.5 \mathrm{kpc}$ from the Galactic center, in the spherically symmetric self-similar model. Thus,

$$
v_{n \pm}\left(r_{\odot}\right)=\frac{R}{t} \xi_{n \pm} \frac{d \Lambda}{d \xi}\left(\xi_{n \pm}\right)
$$


where $R=2.4 \mathrm{Mpc}, t=13.7 \mathrm{Gyr}$, and the $\xi_{n \pm}$ are the solutions of:

$$
r_{\odot}=R \Lambda(\xi) \quad .
$$

There are two solutions for each $n$ but the corresponding speeds are nearly equal. The difference between $v_{n+}\left(r_{\odot}\right)$ and $v_{n-}\left(r_{\odot}\right)$ is ignored in Table $\mathrm{V}$.

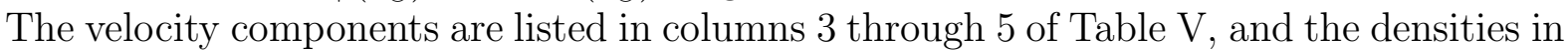
columns 6 and 7. The previous sections provide two ways to estimate the flow velocities and densities. For those flows whose inner caustic rings are close to us, we have the description of Section IIIC. For those flows whose inner caustic rings are far from us, we have the description of Section IIID, and specifically the approximate equations (3.45). In either case, we assume that the Sun is in the symmetry plane $(z=0)$ of the model. This leads to some simplifications.

For $z=0$ Eqs. (3.16), which describe the flow near a caustic ring, have two pairs of solutions, corresponding to $\alpha=0$ and $\eta=0$. For $\alpha=0$ we have:

$$
\begin{aligned}
r=\rho & =a+\frac{1}{2} u\left(\eta-\eta_{0}\right)^{2} \\
v_{z} & =0 \\
v_{\rho}=-\frac{\partial \rho}{\partial \eta} & =-u\left(\eta-\eta_{0}\right)=\mp v \sqrt{2\left(\frac{r}{a}-1\right)} \\
d_{ \pm} & =\frac{1}{r} \frac{d M}{d \Omega d t} \frac{1}{2 b \sqrt{r-a}|\sqrt{r-a} \pm \sqrt{p}|} .
\end{aligned}
$$

The choice of sign in the equation for $v_{\rho}$ is independent of the choice of sign in the equation for $d_{ \pm}$. Eqs. (5.3) describe the in and out flows which exist in the plane of the ring for $r>a$. For $\eta=0$ we have

$$
\begin{gathered}
r=\rho=a+p-\frac{1}{2} s \alpha^{2} \\
v_{z}=-\frac{\partial z}{\partial \eta}=-b \alpha=\mp b \sqrt{\frac{2}{s}(a+p-r)} \\
v_{\rho}=-\frac{\partial \rho}{\partial \eta}=+u \eta_{0}= \pm v \sqrt{\frac{2 p}{a}} \\
d_{ \pm}=\frac{1}{r} \frac{d M}{d \Omega d t} \frac{\cos \sqrt{\frac{2}{s}(a+p-r)}}{2 b(a+p-r)} .
\end{gathered}
$$

The choice of sign in the equation for $v_{z}$ is independent of the choice of sign in the equation for $v_{\rho}$. Eqs. (5.4) describe the up and down flows which exist in the plane of the ring for $r<a+p$.

For $z=0$ Eqs. (3.45) become

$$
\begin{array}{rlrl}
\vec{v} & =\hat{\varphi} v \frac{r}{a} \pm \hat{z} v \sqrt{1-\left(\frac{r}{a}\right)^{2}} & & \text { for } r<a \\
& =\hat{\varphi} v \frac{a}{r} \pm \hat{\rho} v \sqrt{1-\left(\frac{a}{r}\right)^{2}} & & \text { for } r>a \\
d_{ \pm} & =\frac{1}{v} \frac{d M}{d \Omega d t} \frac{1}{\left|r^{2}-a^{2}\right|} . &
\end{array}
$$


The (only) justification for Eqs. (3.45), and hence for Eqs. (5.5), is that they provide a smooth interpolation of the flows between the inner and outer caustics in the limit where the transverse dimensions, $p$ and $q$, of the inner caustic are neglected. For flows of $n$ near 5, whose caustic rings are near us, Eqs. (5.3) and (5.4) provide a more accurate description but they involve the parameters $b$ and $s$ about which we have little information other than that $b$ is of order $v$, and $s$ of order $a$. Eqs. (3.45) and (5.5) merely assume $b=v$ and $s=a$. One may verify that, for $b=v$ and $s=a$, Eqs. (5.5) are consistent with Eqs. (5.3) and (5.4) when $r$ is near $a$ but $p=0$.

The velocity components listed in columns 3 through 5 of Table $\mathrm{V}$ were obtained using Eqs. (5.5). For those values of $n$ (i.e. $n=3,5$ through 14) for which there is evidence for caustic rings of dark matter in the form of sharp rises in the Galactic rotation curve, we used the caustic ring radii $r_{1, n}$ inferred from the positions of the rises. The $r_{1, n}$ are listed in Table IV. For the other values of $n$ (i.e. $n=1,2,4,15$ through 20) we used the predicted values listed in Table III.

Eqs. (5.5) predict that $v_{z}^{n \pm}\left(\vec{r}_{\odot}\right)=0$ for $n \geq 5$ and $v_{\rho}^{n \pm}\left(\vec{r}_{\odot}\right)=0$ for $n \leq 4$. That $v_{z}^{n \pm}\left(\vec{r}_{\odot}\right)=$ 0 for $n \geq 5$ follows from the symmetry of the model. We have set the corresponding entries equal to zero in Table $\mathbb{\nabla}$. On the other hand, that $v_{\rho}^{n \pm}\left(\overrightarrow{r_{\odot}}\right)=0$ for $n \leq 4$ is a consequence of the approximations that led to Eqs. (3.45), in particular the approximation $p=0$. We may compare the zero value for $v_{\rho}$ in Eqs. (5.5) for $r<a$ with the expression for $v_{\rho}$ in Eqs. (5.4) in terms of quantities $v$ and $a$, which we believe we know well, and $p$ on which there is observational information. The widths $p_{n}$ of those caustic rings for which there is evidence in the form of sharp rises in the Galactic rotation curve may be obtained from Table IV by setting $p_{n}=r_{2, n}-r_{1, n}$. For this set, the ratio $\frac{p}{a}$ varies from 0.015 to 0.10 , with an average of 0.05. Eqs. (5.4) imply therefore that, at least for $r$ near $a$, the up and down flows for $r<a+p$ have $\left|v_{\rho}\right| \sim 0.3 v$, whereas Eqs. (5.5) would predict a zero value. As a reminder of these uncertainties, the entries in Table IV for $v_{\rho}^{n \pm}\left(\vec{r}_{\odot}\right)$ with $n<5$ have been left blank.

The flow densities listed in the last two columns of Table $\nabla$ were calculated using Eqs. (5.5) for $n \leq 4$ and $n \geq 10$, and using Eqs. (5.3) with $b=v$ for $5 \leq n \leq 9$. Again we used the observed values $r_{1, n}$ of the caustic ring radii for $n=3,5$ through 14 and the predicted $a_{n}$ for $n=1,24$ and 15 through 20 . The values of $\frac{d M}{d \Omega d t}$ were taken from the last column of Table III. Since the ratio $\frac{v}{b}$ was set equal to one, whereas it is only known to be of order one, all density estimates are uncertain by at least a factor two.

\section{SUMMARY}

In this paper our main goal has been to provide a complete and self-contained description of the caustic ring model of the Milky Way halo, so that anyone wishing to compare the model with observations may readily obtain its predictions. Aside from the assumption of cold collisionless dark matter, the defining properties of the model are axial and reflection symmetry, self-similarity and net overall rotation. The model is a proposal for the full phase space distribution of the Milky Way halo, which is described as a set of discrete flows with stated densities and velocities everywhere.

In Section II, we discussed the general conditions under which the time evolution of a self-gravitating cold collisionless fluid is self-similar. We found that self-similarity does not require symmetry. We discussed three cases: no symmetry, spherical symmetry, and axial symmetry. We derived the equations of motion for the reduced variables in each instance, and the initial conditions consistent with self-similarity. 
The model breaks spherical symmetry because the dark matter particles carry angular momentum. The angular momentum distribution determines the properties of the inner parts of the halo, and in particular of the inner caustics. However, on large scales, the model is nearly spherically symmetric. The spherically symmetric model was originally described by Fillmore and Goldreich [7], and by Bertschinger [8]. It depends on one dimensionless parameter $\epsilon$ and two dimensionful parameters. The latter merely set the scale of the halo's extent in phase space. They are determined in our model by requiring the Galactic rotation velocity at the location of the Sun to be $220 \mathrm{~km} / \mathrm{s}$ and the age of the Galaxy to be $13.7 \mathrm{Gyr}$. The parameter $\epsilon$ is related to the slope of the power spectrum of density perturbations on galactic scales. This implies $\epsilon \sim 0.3$. The properties of the model fitted to observation do not depend sharply on $\epsilon$. We set $\epsilon=0.3$ for definiteness.

The properties of the outer caustic spheres, the flow speeds and the mass infall rates were calculated in the spherically symmetric model. The properties of the first twenty outer caustics are listed in Table II. The flow speeds at the position of the Sun are listed in Table V. The flow speeds at the location of each flow's inner caustic are listed in Table III. The mass infall rates are given by Eq. (3.41). Their values at the inner caustic radii are given in Table IIII.

The structure of the inner caustics depends on the angular momentum distribution of the infalling dark matter particles. The additional parameters introduced in our axially symmetric and self-similar halo model, relative to the spherically symmetric model, are the components $\nu_{r}(\alpha), \nu_{\theta}(\alpha)$ and $\nu_{\varphi}(\alpha)$ of the rescaled initial velocity distribution on the turnaround sphere, Eq. (2.36). The radial component $\nu_{r}(\alpha)$ only plays a minimal role [9] because it does not contribute to angular momentum. We assume that the angular momentum distribution is such that there is net overall rotation, i.e. $\nu_{\varphi}(\alpha)$ dominates over $\nu_{\theta}(\alpha)$. We make this assumption because in that case the inner caustics are tricusp rings. There is evidence for such rings in the Milky Way and other isolated spiral galaxies. The evidence is summarized in Section IV. The radii of the caustic rings depend on a single parameter $j_{\max } \equiv \nu_{\varphi}(0)$. The evidence is consistent with $j_{\max }=0.186$, the value adopted here. The caustic ring radii are listed in Table III.

The flow velocities and densities in the neighborhood of a caustic ring are described in Section IIIC in terms of 6 parameters which may be chosen to be: the caustic ring radius $a_{n}$, the flow speed $v_{n}$ at the caustic, the mass infall rate $\left.\frac{d M}{d \Omega d \eta}\right|_{n}$ there, the time scale $\eta_{0, n}$ over which the particles traverse the caustic, a parameter $b_{n}$ of order $v_{n}$, and a parameter $s_{n}$ of order $a_{n}$. The model values of the first three parameters are given in Table III for the first twenty inner caustics. The last three parameters $\left(\eta_{0, n}, b_{n}\right.$ and $\left.s_{n}\right)$ are related to relatively subtle details of the initial velocity distribution, as described in subsection III.C.4. They determine the transverse sizes $p_{n}$ and $q_{n}$ of the caustic rings (see Eq. (3.19)), and also enter the formula for the density Eq. (3.17).

The model properties are obtained by solving a couple of equations (e.g. Eq. (3.22) on a computer. However, quick estimates can be gotten by using the method of adiabatic invariants. The main results of that method are stated in Eqs. (3.46) and (3.47). The method of adiabatic invariants usually agrees with the results of numerical integration to within $10 \%$ or $20 \%$. It allows estimates of the model properties for $\epsilon \neq 0.3$.

Table $\mathrm{V}$ lists the predicted densities and velocity vectors of the first forty flows on Earth. Knowledge of the local dark matter velocity distribution is essential when interpreting a signal in a dark matter detector on Earth. A striking property of the model is the existence on Earth of a "Big Flow", entry 5+ in Table $\nabla$, whose existence should become readily 
apparent if a signal is found. The model makes predictions for all approaches to the dark matter problem, including direct searches for axions [28, 31] and WIMPs [31, 32], strong and weak gravitational lensing [33], WIMP annihilation in the Galactic halo [34] and the cosmic rays produced thereby [35].

\section{Acknowledgments}

We are grateful to Igor Tkachev for having made available to us his numerical codes for solving the equations of the self-similar model. This work was supported in part by the U.S. Department of Energy under grant DE-FG02-97ER41209. P.S. gratefully acknowledges the hospitality of the Aspen Center for Physics while working on this project. 
TABLE I: The dimensionless rotation velocity squared at the Sun's position $\nu^{2}\left(\epsilon, \frac{r_{\odot}}{R}\right)$, the turnaround radius $R$ and the total mass $M$ for different values of $\epsilon$.

\begin{tabular}{|c|c|c|c|}
\hline$\epsilon$ & $\nu^{2}\left(\epsilon, \frac{r_{\odot}}{R}\right)$ & $R(\mathrm{Mpc})$ & $M\left(M_{\odot}\right)$ \\
\hline 0.1 & 0.21 & 6.0 & $3.1 \times 10^{14}$ \\
0.15 & 0.51 & 3.9 & $8.4 \times 10^{13}$ \\
0.2 & 0.81 & 3.1 & $4.3 \times 10^{13}$ \\
0.25 & 1.08 & 2.7 & $2.8 \times 10^{13}$ \\
0.3 & 1.35 & 2.4 & $2.0 \times 10^{13}$ \\
0.35 & 1.62 & 2.2 & $1.5 \times 10^{13}$ \\
0.4 & 1.91 & 2.0 & $1.2 \times 10^{13}$ \\
0.45 & 2.15 & 1.9 & $9.8 \times 10^{12}$ \\
\hline
\end{tabular}

TABLE II: The radii $R_{n}$ and fold coefficients $A_{n}$ of the first 20 outer caustics.

\begin{tabular}{|c|c|c|}
\hline$n$ & $R_{n}(\mathrm{kpc})$ & $A_{n}\left(M_{\odot} / \mathrm{pc}^{\frac{5}{2}}\right)$ \\
\hline 1 & 436 & $7.7 \times 10^{-4}$ \\
2 & 260 & $8.8 \times 10^{-4}$ \\
3 & 187 & $9.6 \times 10^{-4}$ \\
4 & 147 & $1.02 \times 10^{-3}$ \\
5 & 121 & $1.07 \times 10^{-3}$ \\
6 & 103 & $1.12 \times 10^{-3}$ \\
7 & 89 & $1.16 \times 10^{-3}$ \\
8 & 79 & $1.20 \times 10^{-3}$ \\
9 & 71 & $1.24 \times 10^{-3}$ \\
10 & 64 & $1.27 \times 10^{-3}$ \\
11 & 59 & $1.29 \times 10^{-3}$ \\
12 & 54 & $1.32 \times 10^{-3}$ \\
13 & 50 & $1.35 \times 10^{-3}$ \\
14 & 47 & $1.37 \times 10^{-3}$ \\
15 & 44 & $1.40 \times 10^{-3}$ \\
16 & 41 & $1.42 \times 10^{-3}$ \\
17 & 39 & $1.44 \times 10^{-3}$ \\
18 & 37 & $1.46 \times 10^{-3}$ \\
19 & 35 & $1.48 \times 10^{-3}$ \\
20 & 34 & $1.50 \times 10^{-3}$ \\
\hline
\end{tabular}

[1] C.L. Bennett et al., Ap. J. Suppl. 148 (2003) 1.

[2] P. Sikivie and J. Ipser, Phys. Lett. B291 (1992) 288.

[3] A. Natarajan and P. Sikivie, Phys. Rev. D72 (2005) 083513.

[4] A.G. Doroshkevich et al., MNRAS 192 (1980) 321; A.A. Klypin and S.F. Shandarin, MNRAS 204 (1983) 891; J.M. Centrella and A.L. Melott, Nature 305 (1983) 196; A.L. Melott and S.F. 
TABLE III: The radii $a_{n}$, flow speeds $v_{n}$ and infall rates $\left.\frac{d M}{d \Omega d \eta}\right|_{n}$ for the first 20 inner caustics.

\begin{tabular}{|c|c|c|c|}
\hline$n$ & $\begin{array}{c}a_{n} \\
(\mathrm{kpc})\end{array}$ & $\begin{array}{c}v_{n} \\
(\mathrm{~km} / \mathrm{s})\end{array}$ & $\begin{array}{c}\left.\frac{d M}{d \Omega d \eta}\right|_{n} \\
\left(\frac{M}{\text { sterad·yr }}\right)\end{array}$ \\
\hline 1 & 40.1 & 517 & 53 \\
2 & 20.1 & 523 & 23 \\
3 & 13.6 & 523 & 14 \\
4 & 10.4 & 523 & 10 \\
5 & 8.4 & 522 & 7.8 \\
6 & 7.0 & 521 & 6.3 \\
7 & 6.1 & 521 & 5.3 \\
8 & 5.3 & 520 & 4.5 \\
9 & 4.8 & 517 & 3.9 \\
10 & 4.3 & 515 & 3.4 \\
11 & 4.0 & 512 & 3.1 \\
12 & 3.7 & 510 & 2.8 \\
13 & 3.4 & 507 & 2.5 \\
14 & 3.2 & 505 & 2.3 \\
15 & 3.0 & 503 & 2.1 \\
16 & 2.8 & 501 & 2.0 \\
17 & 2.7 & 499 & 1.8 \\
18 & 2.5 & 497 & 1.7 \\
19 & 2.4 & 496 & 1.6 \\
20 & 2.3 & 494 & 1.5 \\
\hline
\end{tabular}

TABLE IV: Galactocentric radii where observed rises in the Milky Way rotation curves start $\left(r_{1, n}\right)$ and end $\left(r_{2, n}\right)$.

\begin{tabular}{|c|c|c|}
\hline$n$ & $r_{1, n}(\mathrm{kpc})$ & $r_{2, n}(\mathrm{kpc})$ \\
\hline 3 & 12.7 & 13.7 \\
5 & 8.28 & 8.43 \\
6 & 7.30 & 7.42 \\
7 & 6.24 & 6.84 \\
8 & 5.78 & 6.01 \\
9 & 4.91 & 5.32 \\
10 & 4.18 & 4.43 \\
11 & 3.89 & 4.08 \\
12 & 3.58 & 3.75 \\
13 & 3.38 & 3.49 \\
14 & 3.16 & 3.25 \\
\hline
\end{tabular}


TABLE V: Velocity vectors $\vec{v}^{n \pm}\left(\vec{r}_{\odot}\right)$ and densities $d_{n}^{ \pm}\left(\vec{r}_{\odot}\right)$ of the first 40 flows at our location in the Milky Way, in galactic coordinates. $\hat{\varphi}$ is in the direction of galactic rotation, $\hat{z}$ is perpendicular to the galactic plane, and $\hat{\rho}$ is in the radial direction. The two flows with velocity vector $\vec{v}^{n \pm}$ have density $d_{n}^{ \pm}$or $d_{n}^{\mp}$, for each $n$ independently. The $v_{\rho}^{n \pm}\left(\vec{r}_{\odot}\right)$ entries for $n \leq 4$ are not predicted but are expected to be relatively small; see text.

\begin{tabular}{|r|c|c|c|c|c|c|}
\hline$n$ & $\begin{array}{c}v^{n \pm}\left(\vec{r}_{\odot}\right) \\
(\mathrm{km} / \mathrm{s})\end{array}$ & $\begin{array}{c}v_{\varphi}^{n \pm}\left(\vec{r}_{\odot}\right) \\
(\mathrm{km} / \mathrm{s})\end{array}$ & $\begin{array}{c}v_{z}^{n \pm}\left(\vec{r}_{\odot}\right) \\
(\mathrm{km} / \mathrm{s})\end{array}$ & $\begin{array}{c}v_{\rho}^{n \pm}\left(\vec{r}_{\odot}\right) \\
(\mathrm{km} / \mathrm{s})\end{array}$ & $\begin{array}{c}d_{n}^{+}\left(\vec{r}_{\odot}\right) \\
\left(10^{-26} \mathrm{gr} / \mathrm{cm}^{3}\right)\end{array}$ & $\begin{array}{c}d_{n}^{-}\left(\vec{r}_{\odot}\right) \\
\left(10^{-26} \mathrm{gr} / \mathrm{cm}^{3}\right)\end{array}$ \\
\hline 1 & 650 & 140 & \pm 635 & $/$ & 0.3 & 0.3 \\
2 & 600 & 250 & \pm 540 & $/$ & 0.8 & 0.8 \\
3 & 565 & 380 & \pm 420 & $/$ & 1.9 & 1.9 \\
4 & 540 & 440 & \pm 310 & $/$ & 3.4 & 3.4 \\
5 & 520 & 505 & 0 & \pm 120 & 150 & 15 \\
6 & 500 & 430 & 0 & \pm 260 & 6.0 & 3.1 \\
7 & 490 & 360 & 0 & \pm 330 & 3.9 & 1.2 \\
8 & 475 & 325 & 0 & \pm 350 & 1.9 & 1.0 \\
9 & 460 & 265 & 0 & \pm 375 & 1.4 & 0.7 \\
10 & 450 & 220 & 0 & \pm 390 & 0.9 & 0.9 \\
11 & 440 & 200 & 0 & \pm 390 & 0.8 & 0.8 \\
12 & 430 & 180 & 0 & \pm 390 & 0.7 & 0.7 \\
13 & 420 & 170 & 0 & \pm 390 & 0.6 & 0.6 \\
14 & 415 & 155 & 0 & \pm 385 & 0.6 & 0.6 \\
15 & 405 & 140 & 0 & \pm 380 & 0.5 & 0.5 \\
16 & 400 & 130 & 0 & \pm 375 & 0.5 & 0.5 \\
17 & 390 & 120 & 0 & \pm 370 & 0.5 & 0.5 \\
18 & 380 & 110 & 0 & \pm 365 & 0.4 & 0.4 \\
19 & 375 & 100 & 0 & \pm 360 & 0.4 & 0.4 \\
20 & 370 & 95 & 0 & \pm 355 & 0.4 & 0.4 \\
\hline
\end{tabular}

Shandarin, Nature 346 (1990) 633.

[5] D. Stiff and L. Widrow, Phys. Rev. Lett. 90 (2003) 211301.

[6] M. Vogelsberger, S.D.M. White, A. Helmi and V. Springel, MNRAS 385 (2008) 236.

[7] J.A. Fillmore, P. Goldreich, Ap. J. 281 (1984) 1.

[8] E. Bertschinger, Ap. J. Suppl. 58 (1985) 39.

[9] A. Natarajan and P. Sikivie, Phys. Rev. D73 (2006) 023510.

[10] P. Sikivie, Phys. Lett. B432 (1998) 139.

[11] P. Sikivie, Phys. Rev. D60 (1999) 063501.

[12] P. Sikivie, Phys. Lett. B567 (2003) 1.

[13] W. Kinney and P. Sikivie, Phys. Rev. D61 (2000) 087305.

[14] P. Sikivie, I. Tkachev, Y. Wang, Phys. Rev. Lett. 75 (1995) 2911; Phys. Rev. D56 (1997) 1863.

[15] S. Perlmutter et al., Ap. J. 517 (1999) 565; A. Riess et al., Astron. J. 116 (1998) 1009.

[16] A.G. Doroshkevitch, Astrophysics 6 (1970) 320; P.J.E. Peebles, Ap. J. 277 (1984) 470; Y. Hoffmann and J. Shaham, Ap. J. 297 (1985) 16.

[17] J.F. Navarro, C.S. Frenk and S.D.M. White, Ap. J. 462 (1996) 563; B. Moore, Ap. J. Lett. 
499 (1998) L5.

[18] K.G. Begeman, A.H. Broeils and R.H. Sanders, MNRAS 249 (1991) 523.

[19] R.H. Sanders, Ap.J. 473 (1996) 117.

[20] D. Zaritsky, R. Smith, C. Frenk and S.D.M. White, Ap.J. 478 (1997) 39; D. Zaritsky, in Galactic Halos: a UC Santa Cruz Workshop Astronomical Society of the Pacific Conference Series Vol. 136 (1998) 233.

[21] P. Fisher et al., Astron. J. 120 (2000) 1198; T.A. McKay et al., astro-ph/0108013 .

[22] J.N. Bahcall and R.M. Soneira, Ap. J. Suppl. 44 (1980) 73; J.A.R. Caldwell and J.P. Ostriker, Ap. J. 251 (1981) 61.

[23] D.P. Clemens, Ap. J. 295 (1985) 422.

[24] D.B. Sanders et al., Ap. J. Suppl. 60 (1986) 1; D.P. Clements et al., Ap. J. Suppl. 60 (1986) 297.

[25] R.P. Olling and M. Merrifield, MNRAS 311 (2000) 361.

[26] H. Newberg et al., Ap. J. 569 (2002) 245; B. Yanny et al., Ap. J. 588 (2003) 824; R.A. Ibata et al., MNRAS 340 (2003) L21; H.J. Rocha-Pinto et al., Ap. J. 594 (2003) L115.

[27] A. Natarajan and P. Sikivie, Phys. Rev. D76 (2007) 023505.

[28] S. Asztalos et al., Phys. Rev. D64 (2001) 092003; L.D. Duffy et al., Phys, Rev, Lett. 95 (2005) 091304 and Phys. Rev. D74 (2006) 012006.

[29] Z. Ahmed et al., astro-ph/0802.3530; J. Angle et al., Phys. Rev. Lett. 100 (2008) 021303; A. Benoit et al., Phys. Lett. B545 (2002) 43; R. Bernabei et al., Int. J. Mod. Phys. D13 (2004) 2127, and arXiv:0804.2741; G.J. Alner et al., Astroparticle Phys. 28 (2007) 287; G. Angloher et al., Astroparticle Phys. 23 (2005) 325.

[30] P. Sikivie, Velocity peaks and caustic rings, astro-ph/9810286, in the Proceedings of the Second International Workshop on the Identification of Dark Matter, edited by N. Spooner and V. Kudryavtsev, World Scientific 1999, p 58.

[31] F.-S. Ling, P. Sikivie and S. Wick, Phys. Rev. D70 (2004) 123503.

[32] J.D. Vergados, Phys. Rev. D63 (2001) 063511; A.M. Green, Phys. Rev. D63 (2001) 103003; G. Gelmini and P. Gondolo, Phys. Rev. D64 (2001) 023504.

[33] C. Hogan, Ap. J. 527 (1999) 42; C. Charmousis, V. Onemli, Z. Qiu and P. Sikivie, Phys. Rev. D67 (2003) 103502; R. Gavazzi, R. Mohayaee and B. Fort, Astron. Astroph. 445 (2006) 43; V. Onemli, Phys. Rev. D74 (2006) 123010 and Int. J. Mod. Phys. D15 (2006) 2059.

[34] L. Bergstrom, J. Edsjo and C. Gunnarsson, Phys. Rev. D63 (2001) 083515; C. Hogan, Phys. Rev. D64 (2001) 063515; L. Pieri and E. Branchini, JCAP 0505 (2005) 007; R. Mohayaee and S. Shandarin, astro-ph/0503163; A. Natarajan, Phys. Rev. D75 (2007) 123514; R. Mohayaee, S. Shandarin and J. Silk, JCAP 0705 (2007) 015; A. Natarajan and P. Sikivie, Phys. Rev. D77 (2008) 043531.

[35] R. Mohayaee and P. Salati, arXiv:0801.3271. 


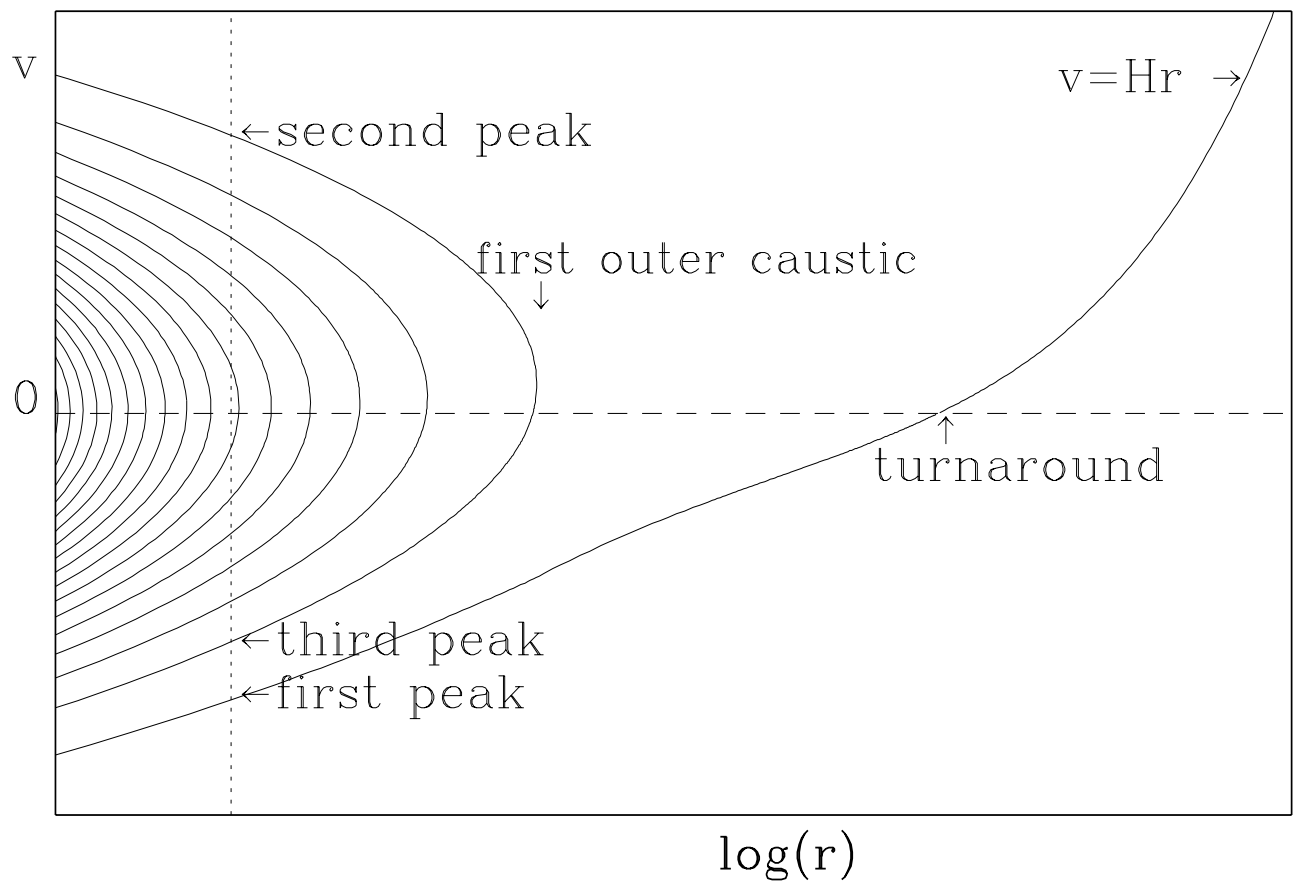

FIG. 1: Qualitative description of the phase space distribution of dark matter particles in a halo at a fixed moment of time. The solid lines represent occupied phase space cells. The vertical dotted line gives the observer position. Each intersection of the solid and dotted lines corresponds to a dark matter flow at the observer position.

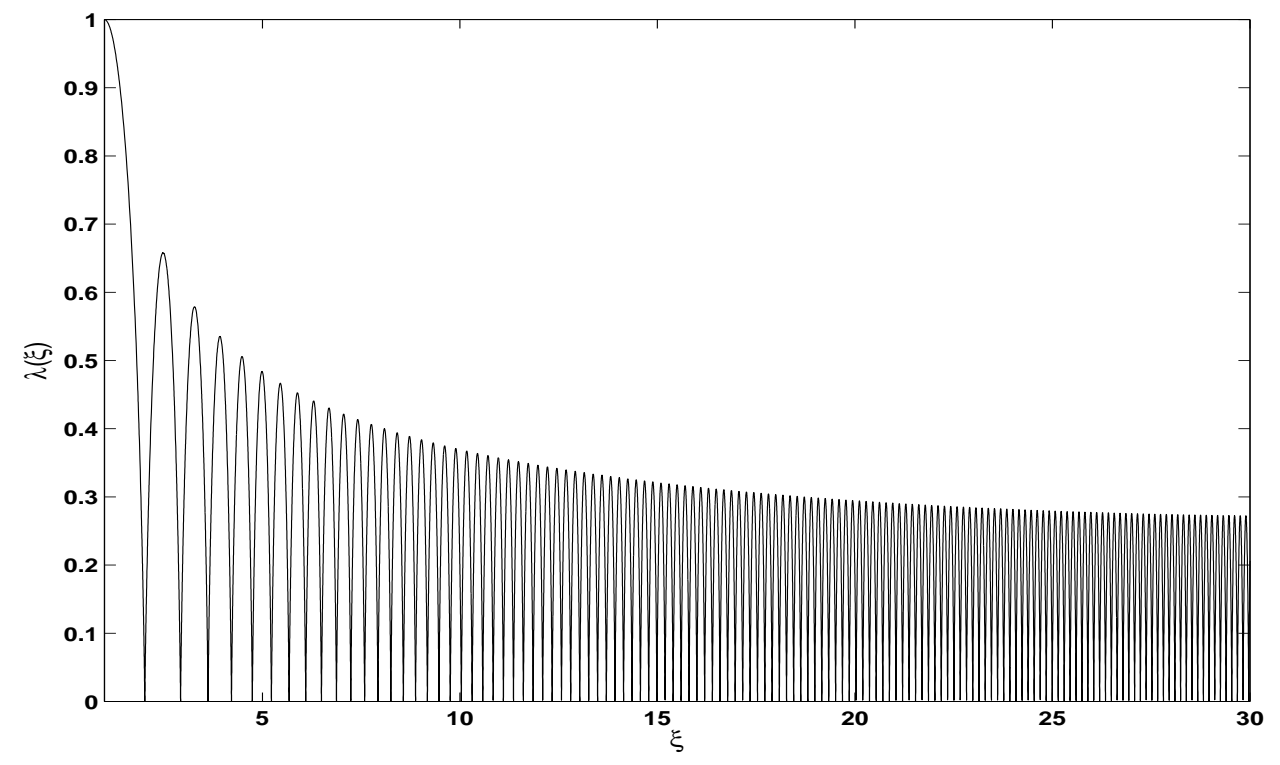

FIG. 2: The function $\lambda(\xi)$ in the spherically symmetric self-similar infall model with $\epsilon=0.3$. 


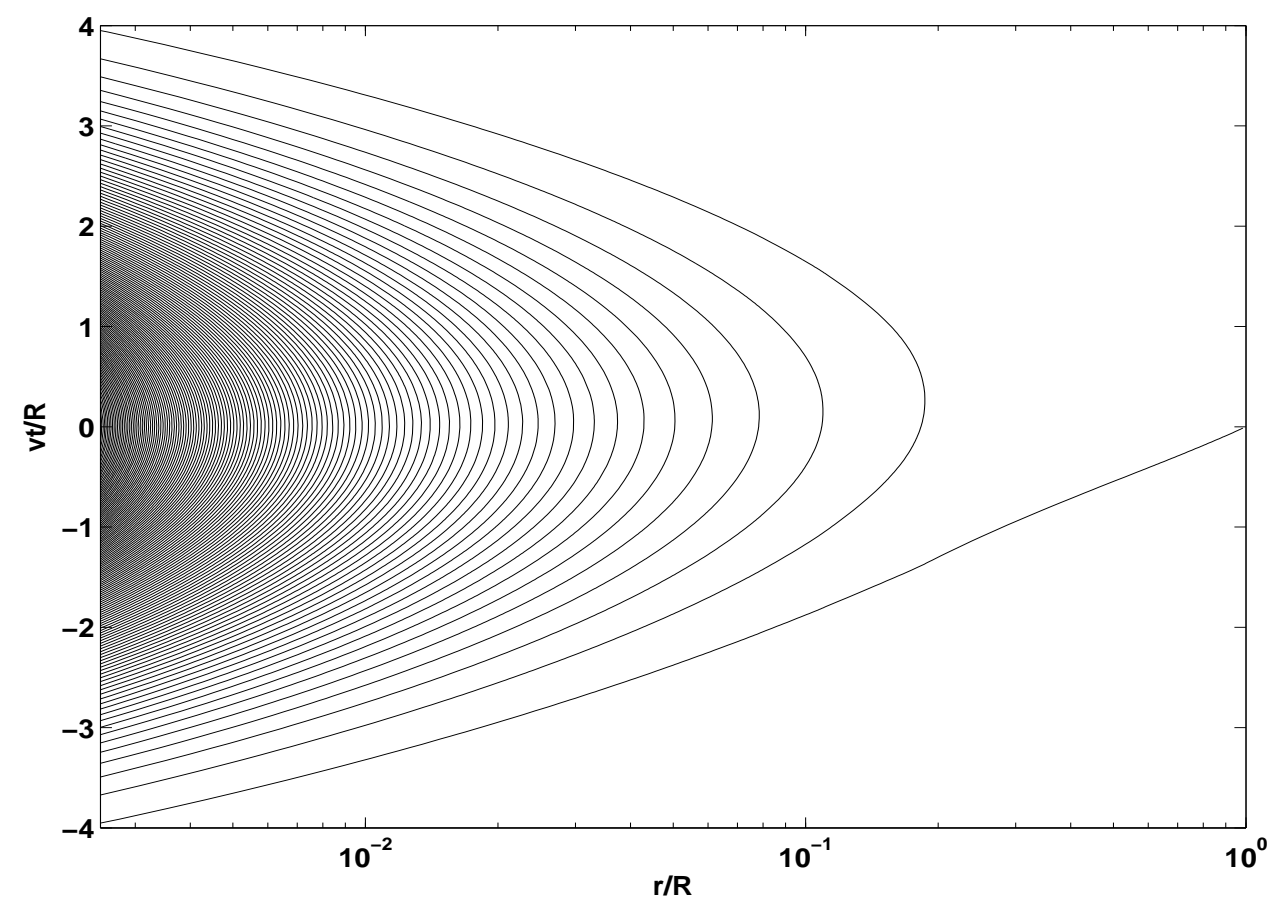

FIG. 3: The phase space distribution of halo dark matter particles at a fixed moment of time in the spherically symmetric self-similar infall model with $\epsilon=0.3$. The solid lines represent occupied phase space cells.

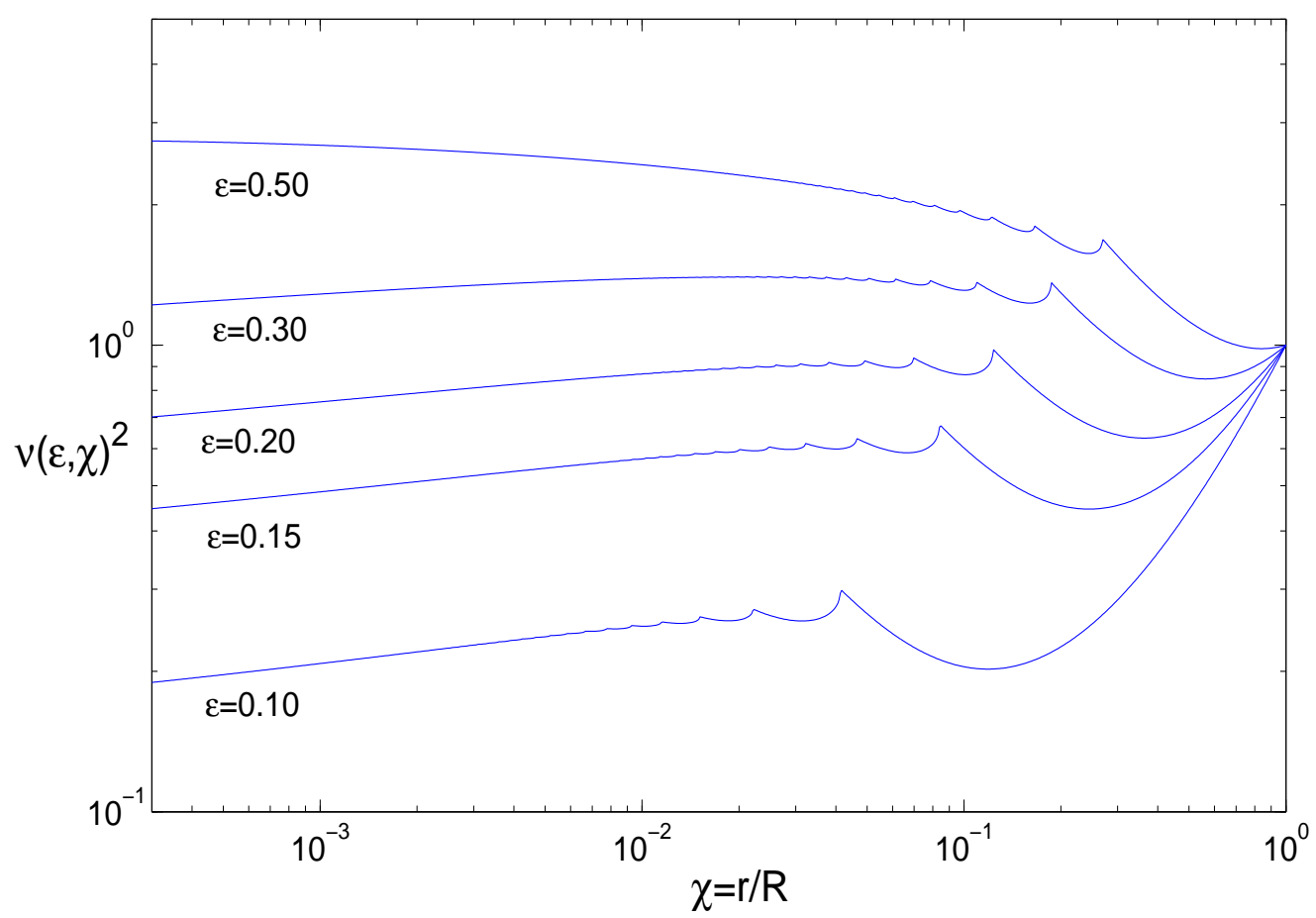

FIG. 4: Rotational velocity squared curves for different values of $\epsilon$, and $j=0$. 


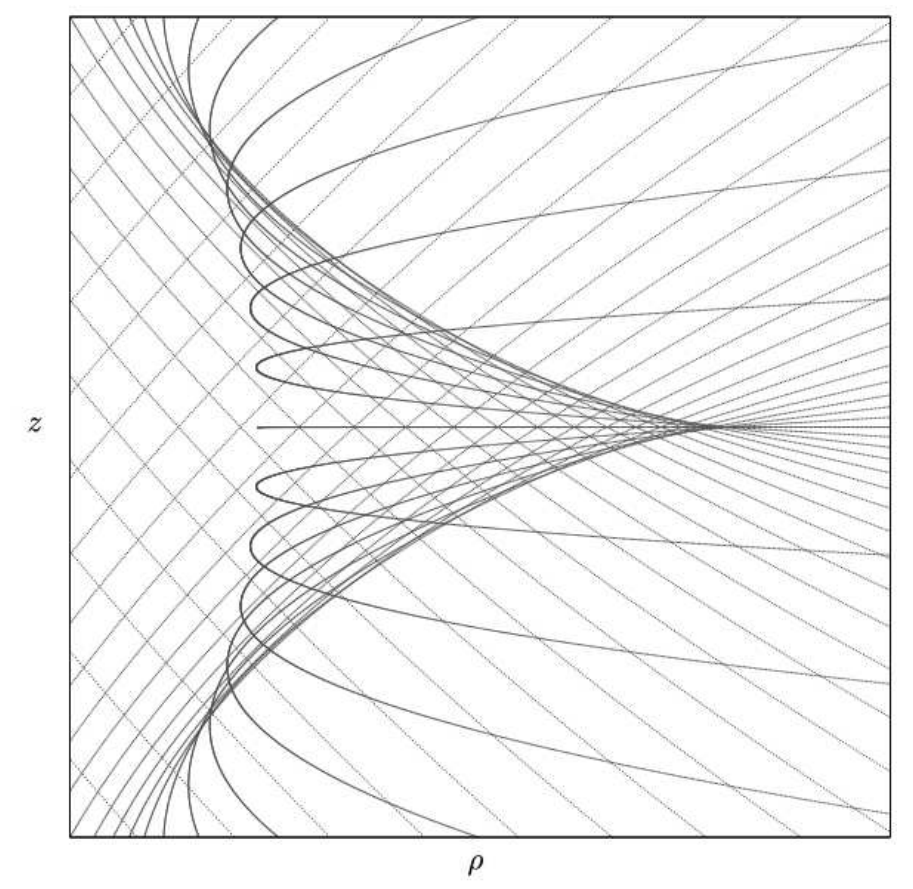

FIG. 5: Dark matter trajectories forming a caustic ring of dark matter, in $\rho-z$ cross-section.

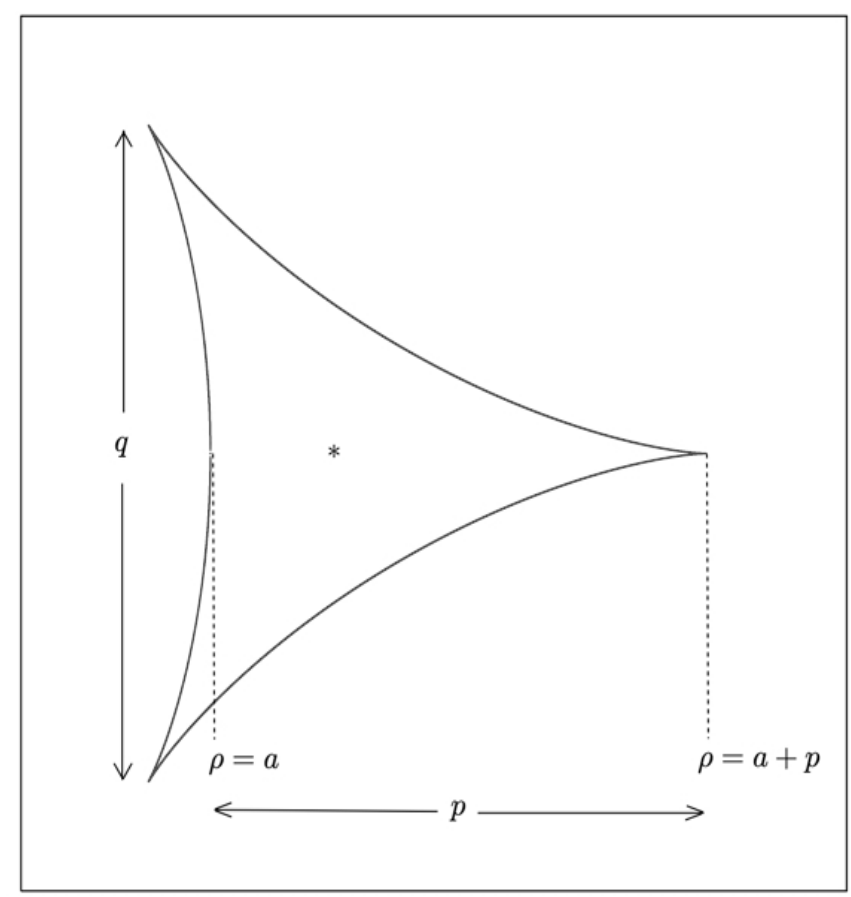

FIG. 6: The envelope of the trajectories that are shown in Fig. 5. The density diverges at the envelope in the limit of zero velocity dispersion. We refer to the shape shown as the "tricusp". What is meant by the radius $a$ and the transverse sizes $p$ and $q$ of a caustic ring is indicated. The tricusp has a discrete symmetry involving a rescaling and rotation by $120^{\circ}$ about the central point, indicated by a star. 